\title{
Development of a CFD methodology for the numerical simulation of irregular sea-states
}

\author{
Anthony Romanowski ${ }^{*}$, Tahsin Tezdogan, Osman Turan \\ Department of Naval Architecture, Ocean and Marine Engineering, Henry Dyer Building, University \\ of Strathclyde, 100 Montrose Street, Glasgow, G4 0LZ, UK \\ * Corresponding author; e-mail: anthony.romanowski@strath.ac.uk, phone: +44(0)1415484532
}

\section{Abstract}

This paper aims to investigate and propose a clear methodology for simulating and maintaining irregular sea simulations within Computational Fluid Dynamics (CFD) for all aspects of the marine industry. As the industry becomes ever more conscious of its overall global emissions, there is an increased interest in beginning to model ever more complex and realistic marine environments. The first step in the beginning to model real-ocean and coastal conditions in CFD is to model irregular seas rather than regular waves. Once this has been achieved further conditions defining realistic oceans can be added, such as varying wind speeds, to these CFD simulations.

To achieve the first step in moving towards realistic ocean simulations, this paper proposes a methodology for meshing and time step calculations for completely unknown irregular seas, along with the best practices for such simulations. The methodology is based upon a preliminary statistical analysis of irregular seas, aiming to break down the irregular sea into key points that will define both the meshing and time step methodologies.

Further to this, example simulations solely focusing on the generated free-surfaces are presented, along with a discussion on the methodology's accuracy and limitations within CFD. These simulations also provide practical data on the modelling and simulating of irregular seas.

Keywords: Computational Fluid Dynamics, Irregular Waves, Significant Wave Height

\subsection{Introduction}

It has been shown that over $90 \%$ of global trade is transported over the sea, thus highlighting the importance the shipping industry plays in a unified global economy (Rahm, 2015). Growing worldwide concern over the volume of emissions and particulate matter (PM) emitted from a ship impacts the brand and corporate responsibility of shipping companies. This is dominantly due to the shipping industry being highly dependent upon the use of fossil fuels to produce the required energy. Due to global use and hunger for fossil fuels, the level of $\mathrm{CO}_{2}$ in the atmosphere has reached its highest in recorded history. This has led both international and regional organisations, such as the International 
Maritime Organisation (IMO) and the European Union (EU), to regulate shipping emissions more stringently.

Further to the release of $\mathrm{CO}_{2}$, it has been estimated that the shipping industry accounts for $8 \%$ of global $\mathrm{SO}_{\mathrm{x}}$ and $15 \%$ of global $\mathrm{NO}_{\mathrm{x}}$ emissions annually (Rahm, 2015). It is also the leading industries in releasing dust and soot particles, due to the use of burning heavy fuels (Rahm, 2015). It estimated that global shipping's greenhouse gas (GHG) emissions will have doubled or tripled in the worst case scenario by 2050 (European Commission , 2013).

By accurately modelling a realistic non-linear sea, vessels and marine structures can be optimised with an aim to enhance their performance. This would result in a longer working life and thus an improved lifetime efficiency of the vessel or structure, helping to reduce the marine industry's global emissions. Two examples of utilising real sea way conditions are put forward below:

1. To reduce the added resistance and increase the accuracy of the calculated results for a large container vessel, with the aim of minimising emissions or enabling an alternate power source to be used.

2. To optimise the motion performance of small vessels vital in supporting offshore industries, thus enabling such vessels to continue working safety in ever more varied sea states (Offshore Wind, 2017).

As technology and computational power increases, the marine industry has begun to utilise Computational Fluid Dynamics (CFD) as a common means to evaluate vessel performance over the past few years. Due to the increasing demand on the industry to minimise GHG emissions, a significant focus has been drawn to evaluating vessels characteristics in real world conditions. However, as technology has only recently allowed such CFD simulations to be conducted, the original benchmark utilised potential flow theories to evaluate such performance in regular or irregular waves. These potential flow methods are limited due to restrictions within these methods, and therefore are restricted in accuracy. Due to the increase in technology and cloud based simulating, it is becoming ever more realistic for industries to consider running such real-world simulations. As there are significant advantages over running multiple regular and calm water simulations.

However, there is limited to no clear literature describing a methodology for simulating and modelling irregular seas with the aim of modelling non-linear sea ways seen in the real-world. It is, therefore, the primary focus of this study to provide a clear and accurate methodology for simulating a non-linear unknown sea way using CFD.

To the best of our knowledge, to date, no studies exist which provide and clear a precise methodology for simulating any irregular sea state within CFD, along with a discussion on the key factors affecting the accuracy of the simulation. 
It has been highlighted by Shen et al. (2014) that the use of Unsteady Reynolds-averaged Navier Stokes (URANS) CFD coupled with irregular seas can achieve accurate results with a limited number of simulations. Within Shen et al.'s (2014) work, a model scale vessel was simulated in long crested irregular seas, although a high accuracy relating to the results was achieved, the methodology and running time constrain the applicability of the research. As the research focused on long crested waves, a significant reduction in compatible hull designs is caused. This is due to long crested irregular seas being most commonly seen in the open ocean with a significant time required for such sea states to develop. In addition, the use of the superposition method to generate free surface limits the complexity that can be seen in truly non-linear sea ways. Ming et al (2013) applied a similar methodology while investigating oblique irregular seas in model scale to derive multiple transfer functions and obtain an even higher definition for the added resistance. Nevertheless, as both methods utilised the superposition method for modelling the free-surface, along with a limited time period and a predetermined freesurface cut, the ability to model a multi order sea way with the methods proposed within these papers fails to be applicable outside of such research.

Therefore, both Ming et al (2013) and Shen et al. (2014) show the advantages of running such simulations to achieve a greater overall picture of the vessels thrust requirements and motion characteristics in real sea conditions. However, both papers do not develop or propose a clear methodology that can be applied in modelling irregular seas and applied within the industry for advanced analysis with ease.

Hence, it is with this knowledge that this study aims at closing this gap and proposing a clear and accurate methodology, for multiple industries, to model and maintain any desired sea state within CFD. It is also hoped that the study will clarify the advantages and disadvantages in modelling irregular seas.

The study below is organised as follows. Section 2 covers the numerical setup for modelling irregular seas is provided, with a preliminary background into the statistical features of irregular seas and how they can be utilised to define the key features in the CFD simulations. From these values, the best practices and definitions for defining and modelling such simulations are provided for use in Star $\mathrm{CCM}+$, along with other applicable CFD solvers. Following this, Section 3 provides a verification study conducted to investigate the ability of the numerical setup to maintain and model the desired sea state. Section 4 provides a discussion on three different sea state simulations, along with both the practical application of such simulations and application within a CFD code, this case being Star CCM+. Finally, Section 5 will provide the overall conclusions drawn from this study, providing an overview of the best practices for defining such simulations and a discussion on the applications within the industry. Future research using the methodology is put forward in this study will be proposed in the final sections of this paper. 


\subsection{Numerical Setup}

Within this section, the key aspects and difficulties in accurately modelling, maintaining and simulating irregular seas will be discussed. In addition, a clear methodology for simulating truly non-linear irregular seas will be proposed based on the statistical and simulated analysis.

As seen from the literature, Computational Fluid Dynamics (CFD) has not yet been extensively used to estimate and model irregular seas for various types of investigations. Thus the literature surrounding a methodology for such a simulation is significantly lacking.

The most commonly used approach to generating irregular seas in the current literature is to use the superposition method with a specified number of waves and respective periods to model a predetermined sea state. This was used to great effect by Shen et al. (2014). However these simulations only encompass a small time period and a prescribed wave pattern. It is therefore aimed with this methodology to provide a set of guidelines to address the issues faced when modelling for truly unknown non-linear irregular seas, and therefore enable a clear representation of real sea states to be modelled.

The diagram in, Figure 1, shows the overall path that is presented in this section, along with the outputs and required user inputs to model a stable simulation. The user inputs are displayed on the left hand side, with key outputs following the path down with only the potential error limits being presented on the right hand side, these will be discussed in greater detail in section 2.1. The path described in Figure 1 is split into three key sections. The initial section focuses on using an in-house MATLAB code to generate a time history of a JONSWAP spectrum defined by the key variables, such as significant wave height $\left(\mathrm{H}_{\mathrm{S}}\right)$ and peak period $\left(\mathrm{T}_{\mathrm{P}}\right)$. The in-house code will continue to post-process the generated time history and produce the key variables used further on in the methodology presented. The methodology described can be easily modified to be used for other spectrums. This analysis provides key wave data that is inputted into the second section which is the meshing and domain definition for the CFD. The final part is the running and monitoring of the simulation. These sections are further elaborated on below, with a primary focus on the two initial setup sections of the path. 


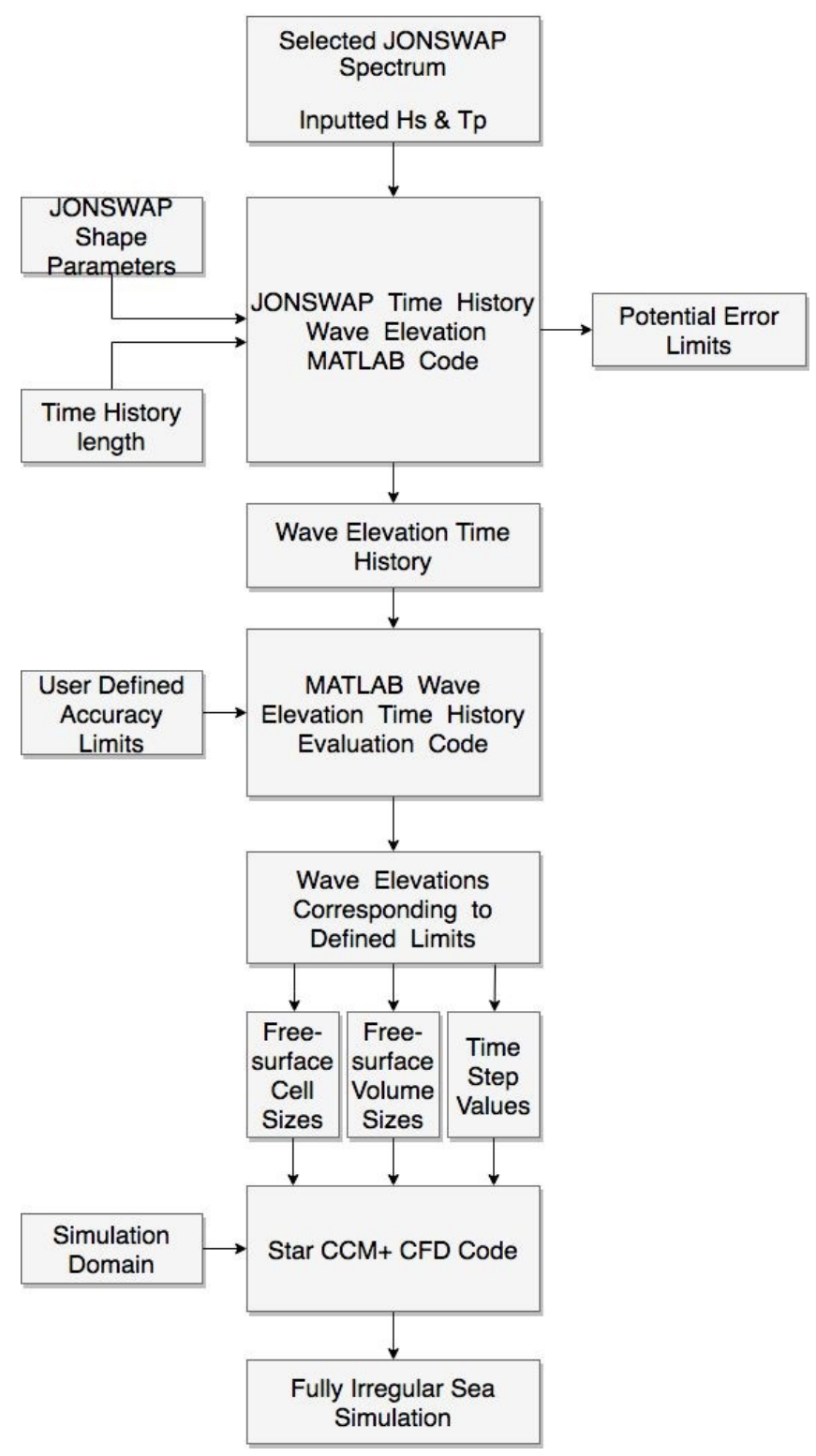

Figure 1 Irregular sea methodology path

\subsection{MATLAB Time History Analysis}

To successfully model and maintain a fully non-linear irregular sea state in CFD, an initial investigation into the observed wave elevations for a given sea state must be conducted. The investigation will provide key data that is used to calculate important input variables that can be implemented into a CFD code later in the methodology. This section will offer a description of the schemes and the in-house MATLAB codes used to develop a time history for the JONSWAP spectrum, along with the approaches used to calculate the various cells sizes, volumes and time step.

As highlighted above, this investigation focuses on the JONSWAP spectrum $\left(\mathrm{S}_{\mathrm{J}}\right)$. However the methodology discussed can be used with any other wave spectrum provided the key steps are followed, 
as shown in Figure 1. To successfully model an unknown sea state within CFD the range of wave elevations must first be found and analysed, this, in turn, will enable the best selection of cell sizes and volumes to be chosen. This is accomplished by coding the desired wave spectrum to produce a time history with respect to wave elevation.

The base equations for the code to define the spectrum were those used within Siemens PLM Star $\mathrm{CCM}+\mathrm{CFD}$ code for generating irregular seas, these definitions were selected to ensure the in-house code, and CFD codes match when generating the time histories, and thus remove a variable when evaluating the methodology discussed later. The basis Pierson-Moskowitz spectrum for the JONSWAP spectrum is given in equation (3), along with the angular spectral peak frequency in equation (2), with $\omega$ defining the wave frequency. The formulation for the JONSWAP spectrum used in this analysis is shown in Eq (3) (CD-Adapco, 2017b), with $\mathrm{H}_{\mathrm{s}}$ defining the significant wave height in metres (m) and $\mathrm{T}_{\mathrm{P}}$ defines the peak period in seconds (s). For which $\mathrm{A}_{\gamma}$ equation (4) is the normalising factor, with $\gamma$ describing the non-dimensional peak shape parameter.

$$
\begin{aligned}
& S_{J}(\omega)=A_{\gamma} S_{P M}(\omega) \gamma^{\exp \left(-0.5\left(\frac{\omega-\omega_{p}}{\sigma \omega_{p}}\right)^{2}\right)} \\
& \omega_{p}=\frac{2 \pi}{T_{p}} \\
& S_{P M}(\omega)=\frac{5}{16}\left(H_{S}^{2} \omega_{p}^{4}\right) \omega^{-5} \exp \left(-\frac{5}{4}\left(\frac{\omega}{\omega_{p}}\right)^{-4}\right) \\
& A_{\gamma}=1-0.287 \ln (\gamma)
\end{aligned}
$$

The in-house code is defined in three sections, the initial section establishes a range of angular frequencies, the second defines the wave spectrum in the frequency domain, in this case, JONSWAP, and the final section converts the defined spectrum into wave elevation with respect to time history. The equations and methodology used to convert the spectrum into a time history are those used by (Branlard, 2010), equations (5), (6) \& (7) show the conversions used, with $\eta(t)$ representing the amplitude with respect to time, $\mathrm{A}_{\mathrm{j}}$ defining the amplitude at a specified frequency and $\varepsilon_{j}$ the randomness factor. An example of a completed time history elevation plot is shown in Figure 2 along with the complete code is shown in Appendix 3 along with the graphical statistical output in Figure 14.

$$
\begin{aligned}
& \eta(t)=\sum_{j=1}^{N} A_{j} \cos \left(2 \pi f_{i}+\varepsilon_{j}\right) \\
& A_{j}=\sqrt{2 S_{J}(f) \Delta f} \\
& \varepsilon_{j}=\operatorname{rand}(0,2 \pi)
\end{aligned}
$$




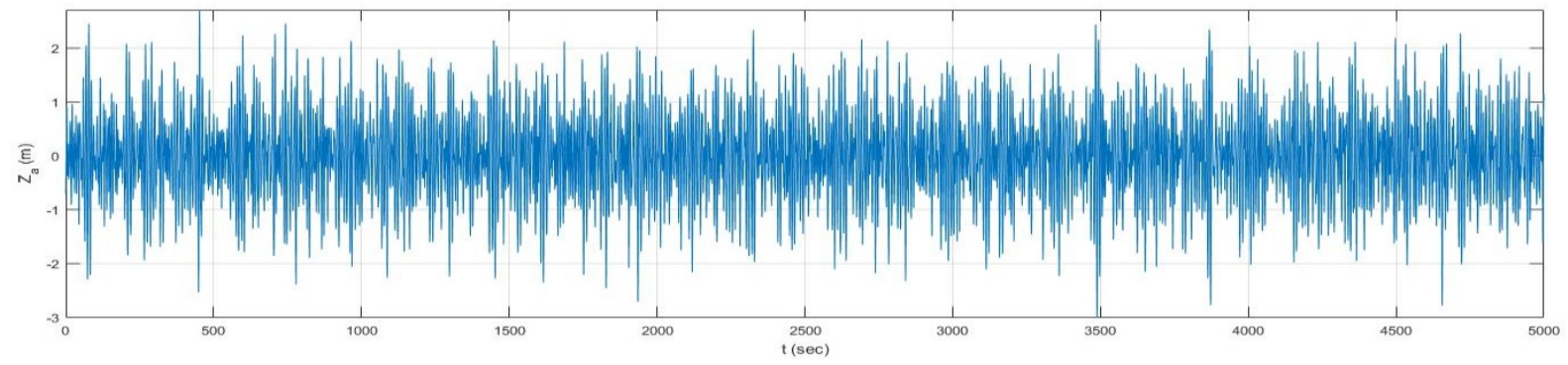

Figure 2 Time history wave elevation plot $(\mathrm{Hs}=3.25 \mathrm{~m} \& \mathrm{Tp}=9.7 \mathrm{~s})$

The raw wave data generated from the in-house code is evaluated in two ways. The first evaluation is applied through a second in-house function which uses the generated wave data, along with a further set of simulated time histories to produce upper and lower bounds of the theoretical potential error seen for a given length of time. This can be key to assessing the completed CFD simulations accuracy, as the simulation time may not correspond to a time history with a calculated significant wave height matching the inputted significant wave height. Figure 3 shows multiple runs of the second in-house function at various lengths of times and the theoretically calculated maximum \& minimum observed error for 1000 sets of time histories. This figure also helps to highlight the importance of having a long simulation time to minimise any observed error, this is discussed in further detail below.

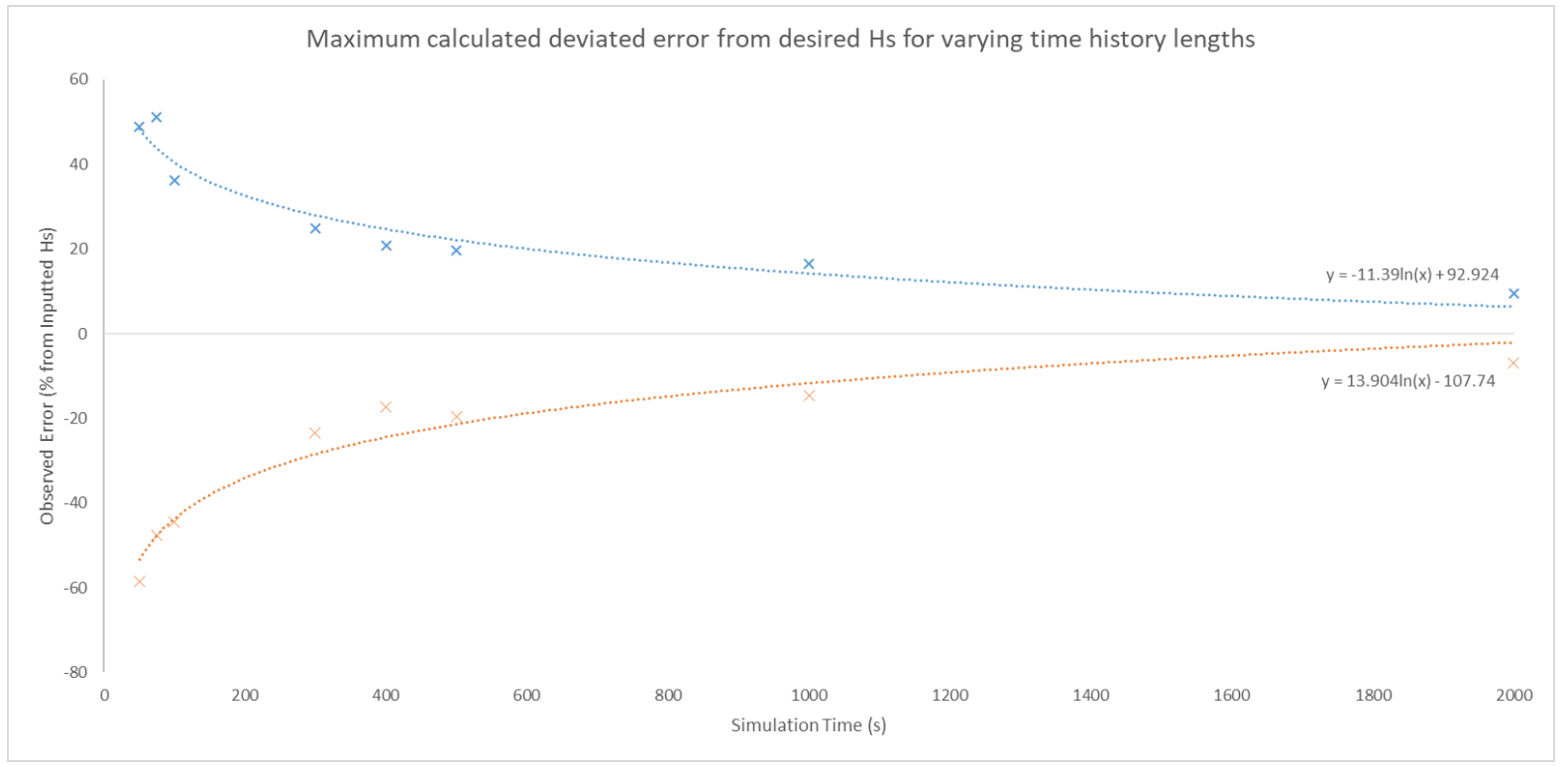

Figure 3 Theoretically calculated error limits from defined Hs with respect to simulated time (SS-5)

As seen from Figure 3, combined with Figure 15, Figure 16, Figure 17, Figure 18 and Figure 19 in Appendix iv, the simulated time histories generated from the in-house code have a consistent pattern. As the theoretical time history length decreases, the maximum error observed increases. This is due to the reduced balance between large and small wave heights and wave lengths. This is a key factor to consider, as the shorter the observed time history for the simulation the greater the potential error will likely be when calculating the significant wave height. This however does not mean that the time history 
did not produce an accurate representation of the desired sea state, just that the time history was too narrower view to cover the whole significant wave height for that sea state. This point, produced through this evaluation, is one of the main reasons why this study will go on to use longer time histories to validate the methodology provided below, to ensure that lower run times will capture all the key characteristics of the simulated sea state.

The second evaluation provides a method to set upper and lower limits on the generated time history wave elevations. This is done by evaluating the distribution of the wave heights and understanding the providence of each wave and its overall effect on desired Hs. This step is the key for an engineer to be able to apply practical knowledge of the type of simulation, and the impact various factors have on the simulation. Based upon the inputs of the user, the upper and lower limits for wave elevation are calculated, these may be for example the maximum and minimum wave elevations observed in a defined irregular seaway, or the upper and lower $5 \%$ of wave heights seen in the time history. The lower limit will correspond to the minimum wave elevation being modelled within the simulation, while the upper limit will define the maximum and minimum heights of the fine free-surface region for mesh generation, this is discussed in further detail below in the mesh generation section.

These limits allow the calculation of key CFD variables along with a further potential error limit within the simulation. An error exceeding the combination of these two evaluations implies that there is a greater problem with the overall simulation, and it must, therefore, be evaluated.

$$
\begin{aligned}
& \mathrm{s}_{\mathrm{p}}=2 \pi \mathrm{H}_{\mathrm{s}} /\left(\mathrm{gT}_{\mathrm{p}}^{2}\right) \\
& s_{m}=2 \pi H_{s} /\left(g T_{z}^{2}\right)
\end{aligned}
$$

These limits are used in partnership with equations (8) \& (9), which have been put forward by Arena et al (2010) to estimate the wave steepness $\left(\mathrm{S}_{\mathrm{P}} \& \mathrm{~S}_{\mathrm{m}}\right.$ wave steepness factors with respect to peak period and zero mean crossing period) in an irregular sea based upon the either the peak period, $\mathrm{T}_{\mathrm{p}}$ equation (8), or the respective zero-up-crossing mean period $\left(T_{z}\right)$ to $S_{m}$ for equation (9). These equations coupled with the recommendations put forward by (CD-Adapco CSP, 2017) and common best practices when modelling waves within $\mathrm{CFD}$, both the $\mathrm{Z}$ and $\mathrm{X}$ lengths for the smallest cell in the free-surface region can be calculated. This is completed all at once within the second in-house code when the time history is being analysed, along with the estimations of various time steps as described in section 3.7. A worked example is shown in Appendix iii for $\mathrm{Hs}=5(\mathrm{~m}), \mathrm{Tp}=12.4$ (s) with key calculated values highlighted in red.

Due to the complexity and non-linear characteristics of an irregular sea way, the mesh refinement volume which defines the smallest cell height should aim to encompass $95 \%$ of the waves, with the extra 5\% covering the tallest waves. The second mesh size control volume has a cell height twice that of the previous volume, this ensures that the waves seen in this volume will be sufficiently refined for. The volume is set to encompass this region mainly due to the non-linearity seen in irregular seas, namely 
small wavelengths and wave heights are not limited to the mean free surface, this can be seen in Figure 4. It is therefore essential in maximising the accuracy of the simulation these waves must be modelled as efficiently as the smallest waves entering the domain. It is for this primary reason that the mesh control volume defining the minimum cell height aims to encompass $95 \%$ of the wave heights generated throughout the simulation, with the second region aiming to capture $99 \%$ of waves and the third domain $99.75 \%$ respectively.

Figure 4 depicts a $2000 \mathrm{~m}$ wave cut, from the simulation previously used as an example, with overlays calculated based on a significant wave height of $5 \mathrm{~m}$ and a peak period of 12.4 seconds. As can be seen, the largest number of waves are encompassed within the first volumetric region, with only the highest waves exceeding the limits of the first region.

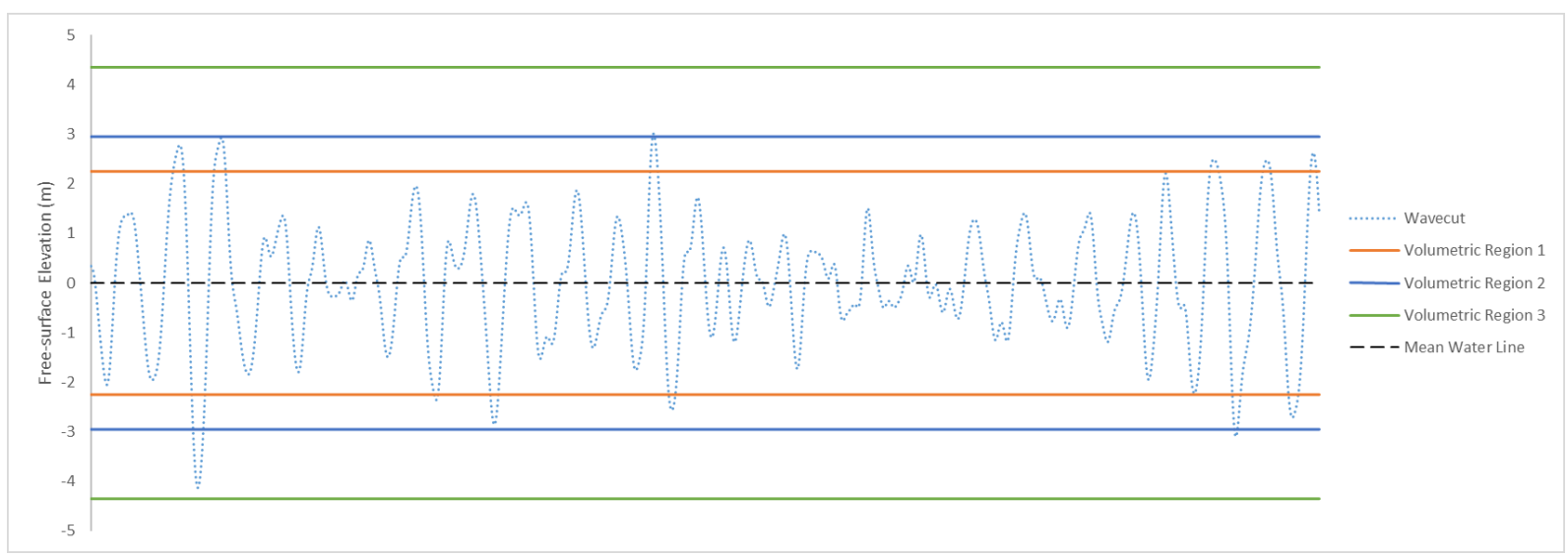

Figure 4 SS-6 Wave cut profile overlaid with calculated refinement volume $Z$ heights, as calculated in Table 10 


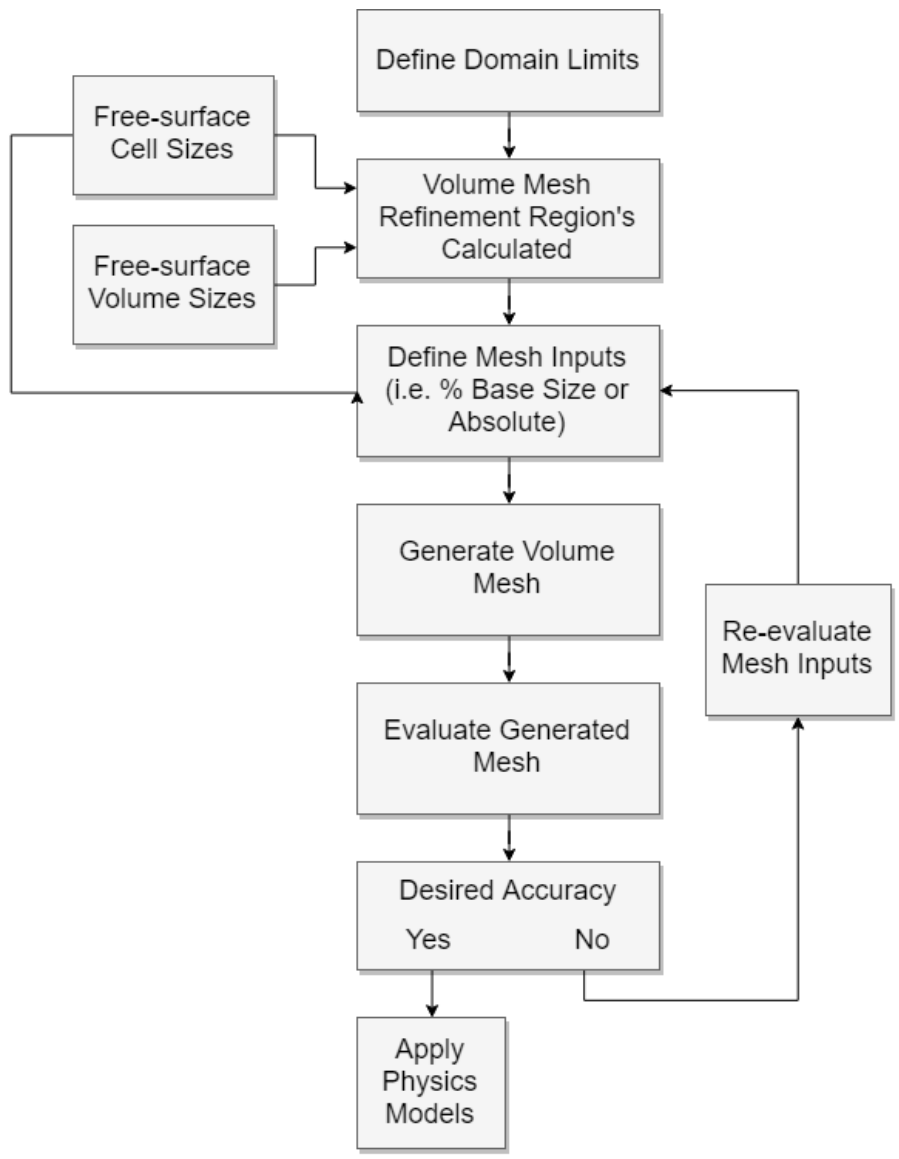

Figure 5 CFD Free-surface mesh setup path with calculated inputs from MATLAB code

\subsection{Domain Size}

To ensure stable simulations and only investigate the effects of varying the mesh strategies, the domain limits were defined by the recommendations proposed by ITTC (2011a). In addition to these recommendations, the best practices advised for use in Star CCM+ (CD-Adapco, 2017b) were also applied. However, it is recommended to apply variuos methods to achieve the best results. Further examples of such reccomendations are seen in Table 1.

Table 1 Boundary locations based upon regular wave simulations for vessels based upon the length between perpendiculars (LBP)

\begin{tabular}{|l|l|l|l|l|l|}
\hline References & Upstream & Down Stream & Up & Down & Transverse \\
\hline (Y.H. Ozdemir, 2014) & 2 LBP & 3 LBP & 2 LBP & 2 LBP & 2 LBP \\
\hline (C.D. Simonsen, 2013) & 0.6 LBP & 2 LBP & Undefined & Undefined & 1.5 LBP \\
\hline
\end{tabular}

\subsection{Mesh Setup}

The mesh setup path for the free-surface is shown in Figure 5. This is in addition to the normal procedures used for modelling the desired mesh in Volume Of Fluid (VOF) CFD simulation while utilising a derivative of the trimmed mesh model. 
To ensure an adequate mesh is generated, the trimmed mesh method should be employed. This method is commonly used for complex mesh problems, with a focus on VOF and free-surface interactions.

To accurately capture all waves to the minimum desired accuracy the cell height and length must be calculated to match the smallest waves that will be modelled. As highlighted above the Minimum Wave Amplitude (MWA) is defined as the lower limit for smallest wave amplitude to be modelled. Based upon the recommendations put forward by Star CCM+, a minimum of 12 cells (CD-Adapco CSP, 2017) should cover the full wave height, defined as the twice MWA. From both the recommendations coupled with the MWA value a minimum cell height can be calculated. Through this investigation, it was found that a minimum of 16 cells per wave height ensures improved stability, in addition to allowing an error margin for the smallest waves created.

The minimum wave height can be further coupled with Equation (8) to estimate the minimum wavelength. This wavelength is used to calculate cell values for both $\mathrm{X} \& \mathrm{Y}$ by applying both the ITTC (2011a), along with Star CCM+ recommendations. It was found that maintaining a constant cell value for both $\mathrm{X} \& \mathrm{Y}$ in the finest mesh refinement region significantly improved simulation stability along with overset mesh interaction. The transvers cell length, Y, may be increased to twice that of the longitudinal in the intervening mesh refinement volumes to reduce the total cell number, however, for best practice, it was found that maintaining a single value for both the longitudinal and transvers proved most effective.

Both the longitudinal and transverse cell lengths may be increased compared with the recommendations to reduce the total cell count. However a maximum aspect ratio between $\mathrm{Z} \& \mathrm{X}$ should not be considered higher than 15 . As significant free surface instabilities will appear and create a diverging simulation.

As seen in Table 10 in Appendix i, key parameters such as the overall domain size, along with the respective sea state characteristics are used to estimate the minimum number of cells within the free surface. By utilising equation (8) the average wave steepness for the specified sea state can be calculated. This coupled with the MWA value found through the preliminary post-processing analysis derives an estimated minimum wavelength. Both the minimum desired wave height to be modelled and the derived minimum wavelength allows a calculated absolute cell height and length (longitudinal and transverse) to be found. An initial mesh number within the first free surface refinement can be estimated based upon the total volume within that refinement and the respective cell volume defined by the calculated X, Y \& Z lengths in the simulations Cartesian coordinate system.

As recommended by Star CCM+ (CD-Adapco, 2017b) a minimum of 8 cells in the $\mathrm{Z}$ height should be used when moving between the various mesh refinement volumes. However, it was found that 8 cells failed to allow complete coverage of a fully developed seaway. Therefore, as each free surface mesh refinement volumes cell height doubles with respect to the previous volume, the number of cells required in the $\mathrm{Z}$ direction to cover $99.5 \%$ of the propagating waves can be calculated. For the case 
shown in Table 10, the volumes upper bounds were defined as $4.35(\mathrm{~m})$ due to the post-processing data showing a wave amplitude of $4.25(\mathrm{~m})$ encompasses $99.65 \%$ of waves seen within the wave cut. This equated to an intermediate cell number of 16 , twice that recommended by Star CCM+. It is vital to note that this value is subject to change depending on the spectrum defined and if any parameters, such as the peak shape parameter, are varied from the predefined JONSWAP spectrum.

As recommended by Star CCM+ along with (Jin, Chai, Duffy, Chin, \& Bose, 2017) the cell growth rate within the domain should be set to such that there are no abrupt or extreme changes in neighbouring cells, ensuring a steady progression from the free surface to the outer domain regions. This helps to mitigate any issues caused by freak waves or wave heights exceeding the limits of the produced in the post-processing of the in-house code.

\subsection{Governing Equations}

The averaged continuity and momentum equations may be written in tensor form and Cartesian coordinates for incompressible flows without body forces as shown below (Ferziger \& Peric, 2002):

$$
\begin{aligned}
& \frac{\partial\left(\rho \bar{u}_{i}\right)}{\partial x_{i}}=0 \\
& \frac{\partial\left(\rho \bar{u}_{i}\right)}{\partial t}+\frac{\partial}{\partial x_{j}}\left(\rho \bar{u}_{i} \bar{u}_{j}+\rho \overline{u_{i}^{\prime} u_{j}^{\prime}}\right)=-\frac{\partial \bar{p}}{\partial x_{i}}+\frac{\partial \bar{\tau}_{i j}}{\partial x_{j}}
\end{aligned}
$$

in which $\tau_{\mathrm{ij}}$ are the mean viscous stress tensor components, as shown in Eq. (12)

$$
\bar{\tau}_{i j}=\mu\left(\frac{\partial \bar{u}_{i}}{\partial x_{j}}+\frac{\partial \bar{u}_{j}}{\partial x_{i}}\right)
$$

and $\mathrm{p}$ is the mean pressure, $\bar{u}_{i}$ is the averaged Cartesian components of the velocity vector, $\rho \overline{u_{i}^{\prime} u_{j}^{\prime}}$ is the Reynolds stresses, $\rho$ is the fluid density and $\mu$ is the dynamic viscosity.

To model fluid flow, the solver employed uses a finite volume method which discretises the integral formulation of the Navier-Stokes equations. A predictor-corrector method is used to link the continuity and momentum equations within the RANS solver.

\subsection{Physics Modelling}

The turbulence model chosen for these simulations and investigations was the Standard K-Epsilon model. This model is extensively used within the industry for multiphase flow and wave modelling, from regular, super position and irregular seas (CD-Adapco, 2017a). 
To ensure accurate modelling of the multiphase flow the use of the 'Volume Of Fluid' (VOF) method has been applied. This method has been used to great effect in modelling both regular and irregular superposition waves by (Tezdogan et al., 2016) and (Shen et al., 2014) respectively. The VOF model key assumption is that the same basic governing equations as those used for a single-phase problem can be solved for all the fluid phases present within the domain, as it is assumed that they will have the same velocity, pressure and temperature.

The second-order convection scheme was applied in all simulations to increase resolution between the interfaces of the different phases.

Although the simulations within this section have not applied any rigid bodies, such as vessels, these can be easily modelled though the use of the Dynamic Fluid Body Interaction (DFBI) model. The model enables full six degrees of motion to be captured through the calculations of the excitation forces and moments acting on the rigid body (CD-Adapco, 2017b).

\subsection{Boundary Conditions}

To ensure stable flow, along with the quickest flow solution, the boundaries must be defined and selected according to recommendations put forward by ITTC (2011a) for similar flow problems.

For flow entering the domain, the use of a velocity inlet over mass flow inlet is recommended, with the opposing boundary defined as a pressure outlet. The respective positioning of inlet and outlet is directly related to the apparent wave and flow heading, best practice relating to such positioning is defined by CD-Adapco (2017b).

Flow problems focused on either head seas or following seas. It is advised to use a symmetry plane (half domain) through the centre, due to the increased mesh numbers seen when meshing. For problems outside of such headings, a full domain should be used.

The top, bottom and outer side may be defined as multiple boundary types, such as velocity inlet, slip wall or symmetry plane. Advice relating to best practice may be seen in CD-Adapco (2017b).

Damping should be applied to both the Outlet and Side, with the application of 'forcing' being used at the Inlet, Side and Outlet boundaries (Royal Institution of Naval Architects, 2018). The lateral component should be forced to zero towards the Side boundaries, with the vertical component forced to zero at the Outlet, thus mitigating any wave reflections and over damping within the domain. Ensuring all wave energy is damped correctly on the pressure outlet and sides is key to maintaining accurate wave propogation and negating any negative wave reflections.

\subsection{Time Step Calculation}

Unlike pure resistance or regular sea simulations, the time step is over refined for the majority of the simulation to ensure the minimum wave modelled for is accounted for in the time step selection. 
Multiple methods for calculating the time step are used. These are primarily based upon the minimum desired wave height, as used in the mesh generation.

Unlike most simulations conducted within this region of research, the Courant Number is not the primary method for estimating the simulations time step. This is not due to its lack of importance, it is due mainly to the time step being focused upon the smallest wave within the domain, and therefore the time step will be significantly lower than normally estimated.

Equation (13) uses the recommendations provided by ITTC (2011a) with an increased power to reduce the time step value to compensate for the smaller waves enterting the domain. However, this method lacks accuracy relating to the specific sea state and the desired accuracy aimed for, as it is based upon regular sea simulations with an increase in power from the recommendation put forward by (ITTC, 2011a), with $T_{\operatorname{Min} \lambda}$ defining the minimum wavelengths period. Therefore, in this work Equations (14), (15) \& (16) were defined to maintain a relationship between a specific sea state and the accuracy desired for.

$$
\begin{aligned}
& \Delta t_{\lambda}=\frac{T_{\operatorname{Min} \lambda}}{2^{9}} \\
& \Delta t_{H}=\frac{0.5 * T_{\operatorname{Min} \lambda}}{2.5 * N Z_{\operatorname{Min} \lambda}} \\
& \omega_{E}=\frac{2 \pi}{T_{\operatorname{Min} \lambda}}-\left(\frac{4 \pi^{2} U}{T_{\operatorname{Min} \lambda}{ }^{2} * g}\right) * \operatorname{Cos}(\theta) \\
& \Delta t_{f e}=\frac{2 \pi}{\omega_{E} 2^{9}}
\end{aligned}
$$

Equation (14) uses the advice provided by Star CCM+ (CD-Adapco, 2017b) to estimate the time step directly relating to the minimum desired wave height and the number of cells in the vertical direction. It is recommended that the free surface should travel no more than $40 \%$ of the cell height per time step. Assuming a constant vertical velocity across half the wavelength from peak to trough an estimated time step can be drawn up relating to the number of cells used for the minimum wave height $\left(N Z_{\operatorname{Min} \lambda}\right)$.

To ensure simulations can be accurately maintained in varying wave headings (with head seas defined as $\theta=180$ ), equations (15) \& (16) are drawn up to calculate the time step based upon the angular encounter frequency $\left(\omega_{E}\right)$, which is calculated with respect to the vessel's speed (U). As in the previous time step calculations the frequency is based upon the minimum desired wave height. 
Equations (13) to (16) highlight the minimum required to maintain a constant and stable free surface. However these time steps may be increased to reduce computational time. It is therefore recommended that a preliminary time step study should be considered.

The use of first-order temporal scheme is advised over second-order to discretise the unsteady term in the Navier-Stokes equations due to potential instability issues arising when coupled with overset regions.

\subsection{Run Time Estimation}

As highlighted above, it is recommended to have a minimum of 300 waves encountered, as defined by ITTC (2011b) for model scale tests and at least 1.5 hours' testing on sea trials. This ensures a large enough range of waves has been encountered to establish an accurate calculation of the significant wave height. As the number of waves encountered reduces, the accuracy of the calculated significant wave height begins to decrease, this can be seen in Figure 3 when comparing the lower and higher run times based on the theoretical margins. This selection of encountering 300 waves can equate to a significantly high run time, with a complex and high mesh number simulation.

This may not be feasible and therefore simulation time may be required to be reduced to become a practically possible. It has been discussed by Mousaviraad (2010) that it is possible to calculate a transfer function for a vessel in an irregular seaway using a single CFD simulation, as highlighted previously. Within the work conducted by Mousaviraad (2010), the time history used only encompasses around a 10 -second period with predefined wavelengths and frequancies. However, by reducing simulation time and cutting the number of waves encountered there is a high potential for errors appearing in the calculated $\mathrm{H}_{\mathrm{s}}$, when limited to such low run times in irregular seas. For Mousaviraad (2010) to overcome the limited time history and calculate the transfer funtions, the application of Discrete Fourier Transform (DFT) was used. This enabled limited time history to be used and based upon to calculate the results.

Therefore, to ensure a stable sea state has occurred, along with the desired Hs, a minimum of 100 waves should be encountered when investigating non-linear irregular seas. This was the minimum value selected for this study, however even a further reduced number of encounter waves may be specified if the mesh and time step definitions match those described above. The key factor to consider when running such low encountered wave simulations is the inaccuracy of the observed significant wave height from the recorded time history. The selection of lower run times should be considered with respect to the patterns observed in Figure 18 as an example. 


\subsection{Verification Study}

In this study the primary focus has been on the free surface mesh refinement and optimising this region for irregular seas. To further verify the work conducted, along with the methodology proposed in the study above, a verification study utilising the methodology devised by Stern et al (2001) has been conducted. As there are no vessels within the study, the verification will focus solely on the free surface refinement and its ability to maintain the inputted significant wave height.

The domain for the verification study was created in mind of having a small vessel, around $25 \mathrm{~m} \mathrm{LOA}$, within it. This is both for reducing the total number of cells required through minimising the domain volume, and verifying a similar sized domain as used in the case study later in this study. The domains length was defined as $1.5 \lambda$ (wave length) of a single regular wave with an equivalent wave height of the desired $\mathrm{H}_{\mathrm{s}}$. All boundaries were defined as stated above.

Each simulation was run with no current or wind, and solely focused on the waves transiting the domain due to their own speed. The simulations were running for a total of 1500 seconds, ensuring around 300 seen waves. Simulating over 300 waves ensures that the calculated Hs will not be distorted due lower run times, as can be estimated from Figure 18. Further to this, each simulation utilised the test setting in the Irregular Wave model within the CFD code, ensuring each simulation had the same incoming wave profile for each simulation. Thus, ensuring the only factor changing would be the grid spacing or time step.

Within this process, a verification study was conducted to investigate the numerical uncertainty with the simulations, $\mathrm{U}_{\mathrm{SN}}$, along with the numerical errors, $\delta_{\mathrm{SN}}$. The numerical errors are assumed to be composed of three elements, the iterative convergence error $\left(\delta_{\mathrm{I}}\right)$, grid-spacing convergence error $\left(\delta_{\mathrm{G}}\right)$ and time-step convergence error $\left(\delta_{\mathrm{T}}\right)$. These, in turn, defines equations $(17) \&(18)$ as proposed by Stern et al (2001) for the numerical error and uncertainty within the simulation.

$$
\begin{aligned}
& \delta_{S N}=\delta_{I}+\delta_{g}+\delta_{t} \\
& U_{S N}^{2}=U_{I}^{2}+U_{G}^{2}+U_{r}^{2}
\end{aligned}
$$

With $\mathrm{U}_{\mathrm{I}}, \mathrm{U}_{\mathrm{G}}$ and $\mathrm{U}_{\mathrm{T}}$ describing the uncertainties for iterative, grid spacing convergence and time step convergence errors respectively.

Due to the transient nature of the simulation, along with the inability to clearly define a point at which a steady state solution has been achieved, the iterative convergence error has been neglect, as it is not suitable for such a simulation and verification study.

From this, both grid spacing and time step convergence studies were conducted according to the Grid Convergence Index (GCI) study proposed by (Roache, 1998). As no fluid stucture interaction would be taking place within these simulations, turbulence model study was not considered. Further to this, other 
studies focusing on wave verification, such as (Lal \& Elangovan, 2008), found no noticable effect on wave elevation due to turbulence model variation. This meant that only the grid spacing and time step variation would have the largest effect on the simulations, and thus are the key focus of this study.

The GCI method was performed with three solutions, each of which had a methodically refined grid spacing or time step. For each solution the primary parameter, i.e. the grid spacing or time step, was coarsened/increased by a specific value, while all other parameters were maintained at a constant value. For example, when conducting the time step convergence study the finest mesh was used for all three simulations and only the time step value was increased. The mesh convergence follows the inverse of this, namely that the smallest time step is used with the grid being coarsened throughout the whole domain by a specific value.

$$
\begin{aligned}
R_{k} & =\frac{\varepsilon_{k 21}}{\varepsilon_{k 32}} \\
\varepsilon_{K 21} & =S_{k 2}-S_{k 1} \\
\varepsilon_{K 32} & =S_{k 3}-S_{k 2}
\end{aligned}
$$

Equation (19) depicts the convergence ratio used to assess the various convergence conditions in the two studies described above. The top term (20) defines the difference between the medium-fine solutions, while the lower term (21) defines the difference between coarse-medium solutions.

Four typical convergence conditions may be seen: (i) monotonic convergence $\left(0<\mathrm{R}_{\mathrm{k}}<1\right)$, (ii) oscillatory convergence $\left(\mathrm{R}_{\mathrm{k}}<0 ;\left|\mathrm{R}_{\mathrm{k}}\right|<1\right)$, (iii) monotonic divergence $\left(\mathrm{R}_{\mathrm{k}}>1\right)$, and (iv) oscillatory divergence $\left(\mathrm{R}_{\mathrm{k}}<0\right.$; $\left|\mathrm{R}_{\mathrm{k}}\right|>1$ ) (Stern et al., 2006).

The generalised Richardson extrapolation is used to predict the uncertainties and numerical error in condition (i). To obtain the uncertainties for condition (ii) a further step must be applied, this is seen in equation (22).

$$
U_{K}=\frac{1}{2}\left(S_{U}-S_{L}\right)
$$

$\mathrm{S}_{\mathrm{U}}$ and $\mathrm{S}_{\mathrm{L}}$ define the maximum and minimum found in the convergence study respectively. Finally, for cases (iii) and (iv), diverging conditions, the uncertainty or the error cannot be assessed (Stern et al. 2006).

As used in similar studies to this work (Terziev, et al., 2018), a uniform refinement ratio $\left(\mathrm{r}_{\mathrm{G}}\right)$ is used to in the mesh convergence study. In the case of this study, the value for $r_{G}$ was defined as $\sqrt{ } 2$ throughout the whole domain and mesh continuum. The final mesh numbers for each of the mesh convergence cases are listed in Table 2. 
Table 2 Final cell numbers for fine, medium and coarse mesh convergence studies

\begin{tabular}{|c|c|c|}
\hline $\begin{array}{c}\text { Mesh } \\
\text { Configuration }\end{array}$ & $\begin{array}{c}\text { Min Z Cell } \\
\text { Height }\end{array}$ & $\begin{array}{c}\text { Cell } \\
\text { Number }\end{array}$ \\
\hline Fine & 0.0219 & $14,798,388$ \\
\hline Medium & 0.0309 & $5,565,638$ \\
\hline Coarse & 0.0438 & $2,105,162$ \\
\hline
\end{tabular}

Table 3 Simulated Hs and relative error from desired value in mesh convergence study

\begin{tabular}{l|c|c}
\cline { 2 - 3 } & $\begin{array}{c}H_{s} \\
\text { (metre) }\end{array}$ & $\begin{array}{c}\text { \% Off Desired } \\
H s\end{array}$ \\
\hline Fine & 5.05 & 0.9901 \\
Medium & 5.485 & 8.8423 \\
Coarse & 5.987 & 16.4857 \\
\hline
\end{tabular}

Following the same method as the mesh convergence study, the time step convergence study used three cases, each of which has their time step increased from the calculated minimum value by a uniform refinement ratio $\left(\mathrm{r}_{\mathrm{T}}\right)$ of 2 . Using equations (13) \& (14) a minimum time step of $0.0035 \mathrm{~s}$ was estimated. The fine mesh setup was used for the time step convergence study.

Utilising equations (19), (20) \& (21), the Richardson coefficient is calculated for both the grid spacing and time step studies. The computed values for both the grid refinement and time step convergence studies can be seen in Table 4 \& Table 5.

The relative errors because of the grid coarsening are seen in Table 3. This preliminary study begins to highlight the effects of grid coarsening upon the desired Hs and in turn the dependency that goes with it.

Table 4 Grid convergence study with respect to Hs

\begin{tabular}{|c|c|c|c|c|c|c|c|c|}
\hline \multirow{2}{*}{ Parameter } & \multirow{2}{*}{$r_{g}$} & \multicolumn{3}{|c|}{ Solutions } & \multirow{2}{*}{$\mathrm{R}_{\mathrm{g}}$} & \multicolumn{3}{|c|}{$\mathrm{GCl}$} \\
\hline & & s1 & s2 & s3 & & $\mathrm{GCl} 21$ & $\mathrm{GCl} 32$ & GCIRatio \\
\hline $\mathrm{Hs}$ & $\sqrt{ } 2$ & 5.050 & 5.485 & 5.987 & 0.870 & 72.058 & 76.257 & 1.058 \\
\hline
\end{tabular}

Table 5 Time-step convergence study with respect to Hs

\begin{tabular}{|c|c|c|c|c|c|c|c|c|}
\hline \multirow{2}{*}{ Parameter } & \multirow{2}{*}{$r_{t}$} & \multicolumn{3}{|c|}{ Solutions } & \multirow{2}{*}{$\mathrm{R}_{\mathrm{t}}$} & \multicolumn{3}{|c|}{$\mathrm{GCl}$} \\
\hline & & s1 & s2 & s3 & & $\mathrm{GCl} 21$ & GCl32 & GCIRatio \\
\hline Hs & 2 & 5.050 & 5.150 & 3.750 & -0.071 & 0.190 & 2.614 & 13.728 \\
\hline
\end{tabular}

As seen in Table 4, a monotonic convergence can be observed from the calculated $\mathrm{R}_{\mathrm{g}}$ value of 0.87 . This was to be expected as through this study it has been made clear that there is a distinct relationship 
between the sustained significant wave height and the inputted value. Table 4 further highlights that such simulations have a dependency upon grid spacing, which in turn directly relates to the predetermined accuracy level being aimed for.

In addition to this, the verification study has further highlighted a dominant part when attempting to maintain a stable free surface, namely the reliance on the time step. Although an oscillatory convergence was seen in the time-step convergence study, represented by $R_{t}$, the free surface failed to maintain a constant and stable surface. Further to this, Table 5 shows a significant dependency upon the time step in maintaining the desired significant wave height, along with a stable simulation. This is noted even further when comparing Figure $6 \&$ Figure 7. Both scalar scenes show the free surface defined by the Volume Fraction of $\mathrm{H}_{2} \mathrm{O}$ in the fine mesh simulation. Figure 6 simulation uses the minimum defined time step, while Figure 7 simulation uses the maximum time step in time-step convergence study. As can be seen from Figure 7, a dominant region of the free surface has broken down and spread across a significant number of cells, causing the defined interface to be lost and trend towards a divergent solution. It is therefore paramount that the time step is selected carefully and only modified with caution.

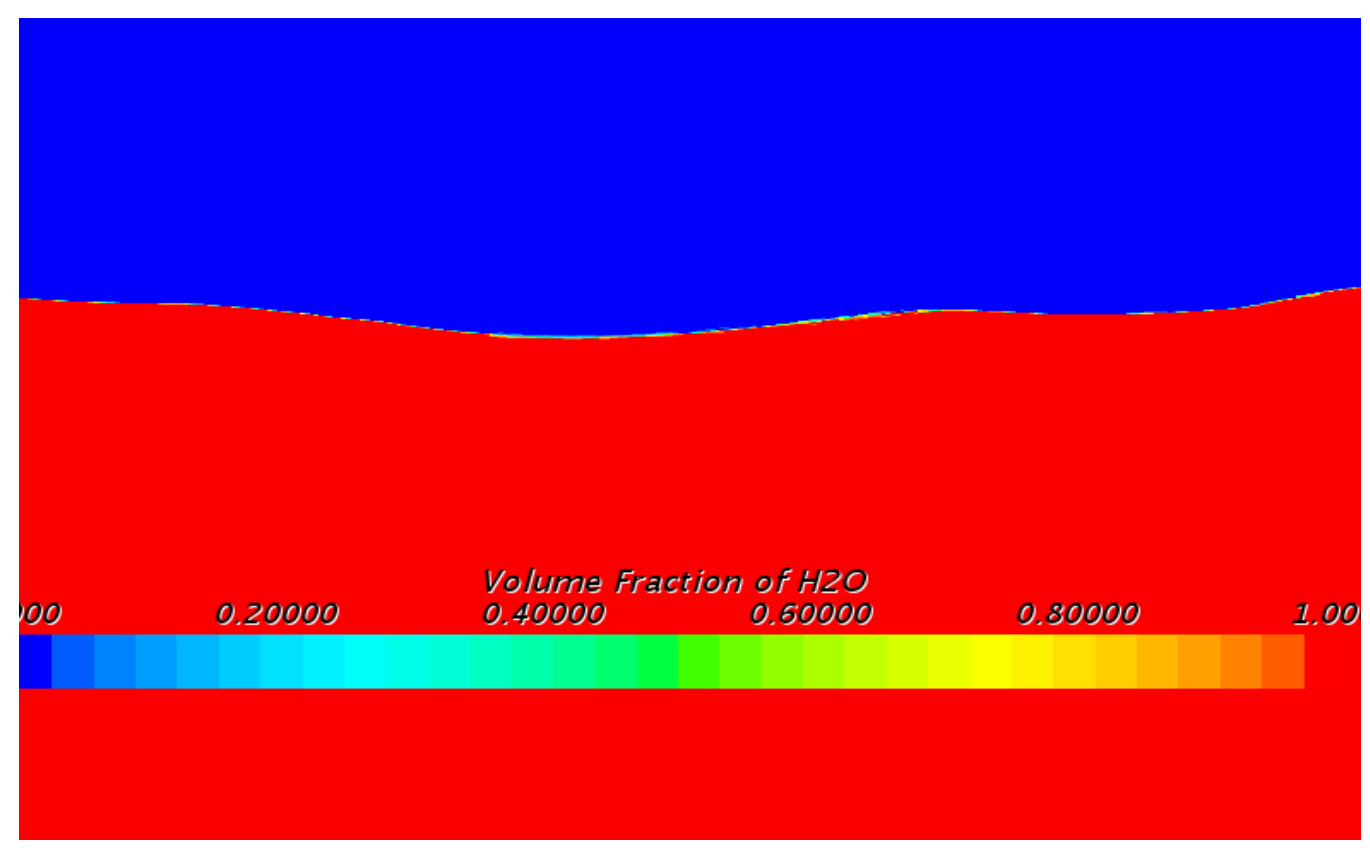

Figure 6 VOF Scalar scene depicting the free surface when $\mathrm{Ts}=\mathbf{0 . 0 0 3 5} \mathrm{s}$ 


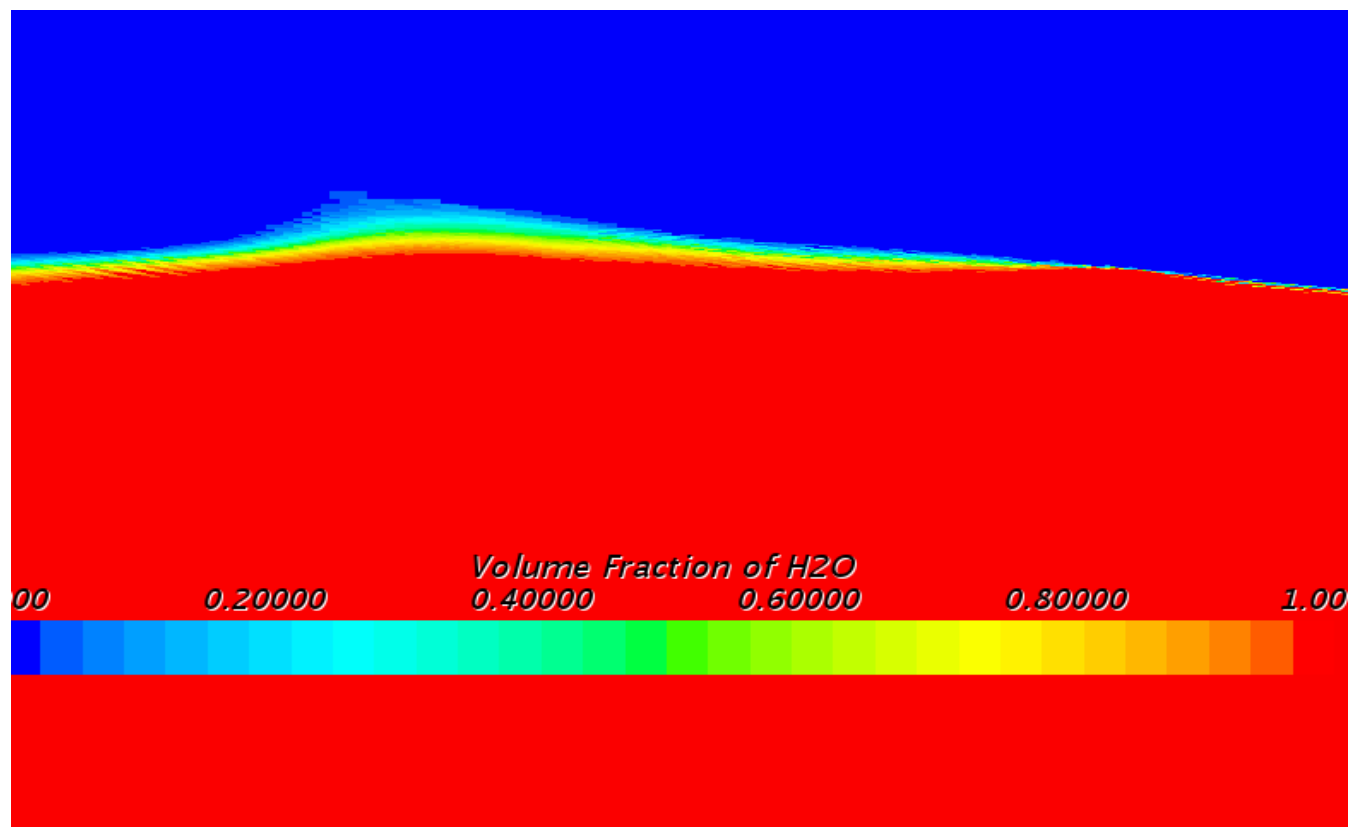

Figure 7 VOF Scalar scene depicting the free surface when $\mathrm{Ts}=0.014 \mathrm{~s}$

\subsection{Results Section}

\subsection{Introduction}

This section will present results and analysis obtained by using the methodology described above for three significant wave heights and mesh accuracy levels. This is carried out to compare the effectiveness of the methodology for various sea states, along with practical limitations of such simulations and methods.

\subsection{Sea State 6}

The initial seta state being discussed in this section will be that also used within the validation and verification section above. As shown above, a significant wave height of $5(\mathrm{~m})$ and a peak period of 12.4 (s) was selected. This has been chosen to show the methodologies accuracy in modelling large sea states that will likely be investigated in CFD for both added resistance and motion analysis.

Based upon these JONSWAP parameters, the initial mesh characteristics and time steps were calculated using the in-house code shown in Appendix ii. The calculated mesh values are shown in Table 6. As can be seen, a minimum cell height of $0.022(\mathrm{~m})$ has been calculated, along with an upper limit of \pm 2.25 (m) from the mean water line for the first refinement region. It can also be seen from Table 6 that a minimum wave height of $0.35(\mathrm{~m})$ will be modelled within this region, based upon a 16 cell distribution in the $\mathrm{Z}$ height. 
Table 6 Calculated cell heights and distributions for a Sea State 6 simulation

\begin{tabular}{|c|r|}
\hline Sea State & 6 \\
$\mathrm{Hs}$ & $5 \mathrm{~m}$ \\
$\mathrm{Tp}$ & $12.4 \mathrm{~s}$ \\
Spectrum & JONSWAP \\
\hline
\end{tabular}

\begin{tabular}{|l|l|}
\hline Av Wave Steepness & 0.0208275 \\
\hline
\end{tabular}

\begin{tabular}{|c|c|c|c|}
\hline $\begin{array}{c}\text { Max Z height in +ve \& -ve } \\
\text { Min Wave Height }\end{array}$ & $\begin{array}{l}2.25 \mathrm{~m} \\
0.35 \mathrm{~m}\end{array}$ & & \\
\hline Free Surface Refinement Region & FrSf_1 & FrSf_2 & FrSf_3 \\
\hline No of Cells per Wave Height & 16 & & \\
\hline \multirow[t]{3}{*}{ Z cell Height } & $0.0219 \mathrm{~m}$ & $0.0438 \mathrm{~m}$ & $0.0875 \mathrm{~m}$ \\
\hline & & $0.7 \mathrm{~m}$ & $1.4 \mathrm{~m}$ \\
\hline & 16 & $2.95 \mathrm{~m}$ & $4.35 \mathrm{~m}$ \\
\hline
\end{tabular}

\begin{tabular}{|c|c|c|c|}
\hline Min Wave length & $16.805 \mathrm{~m}$ & & \\
\hline No of Cells per L & 60 & 4 & 4 \\
\hline X Cell Length & $0.2801 \mathrm{~m}$ & $0.5602 \mathrm{~m}$ & $1.1204 \mathrm{~m}$ \\
\hline Y Cell Length & $0.2801 \mathrm{~m}$ & & \\
\hline
\end{tabular}

Upon the calculation of the various volumes and cell sizes, the simulation was modelled and meshed. Although an initial cell size of $0.022(\mathrm{~m})$ had been calculated, the meshing scheme within the CFD code did not allow a cell of the calculated $\mathrm{Z}$ and $\mathrm{X}$ lengths to be generated. This led to the closest cell to be modelled having a $\mathrm{Z}$ height of $0.024(\mathrm{~m})$. Table 7 shows a comparison between the desired and generated dimensions, along with the potential observed error also being shown. This increased cell height meant that the minimum wave height was split between 15 cells in the $\mathrm{Z}$ height rather than the desired 16 . Due to only a minor increase in cell height, the observed smallest wave was $30 \%$ smaller than the minimum wave height planned for, this can be seen in Figure 8. Figure 9 shows the full wave elevation compared with run time, this figure compared with Figure 10 also highlights the run time limitations as the number of observed waves is significantly lower than can be seen in Figure 9. This backs up the error margins discussed in section 2.1.

Table 7 Comparison of theoretical and generated mesh size ( $\mathrm{Z}$ direction)

\begin{tabular}{|l|l|}
\hline Calculated & Generated \\
\hline $0.022(\mathrm{~m})$ & $0.024(\mathrm{~m})$ \\
\hline Difference & $9.09 \%$ \\
\hline Number of cells per Min Wave Height & $14.6 \sim 15$ \\
\hline
\end{tabular}




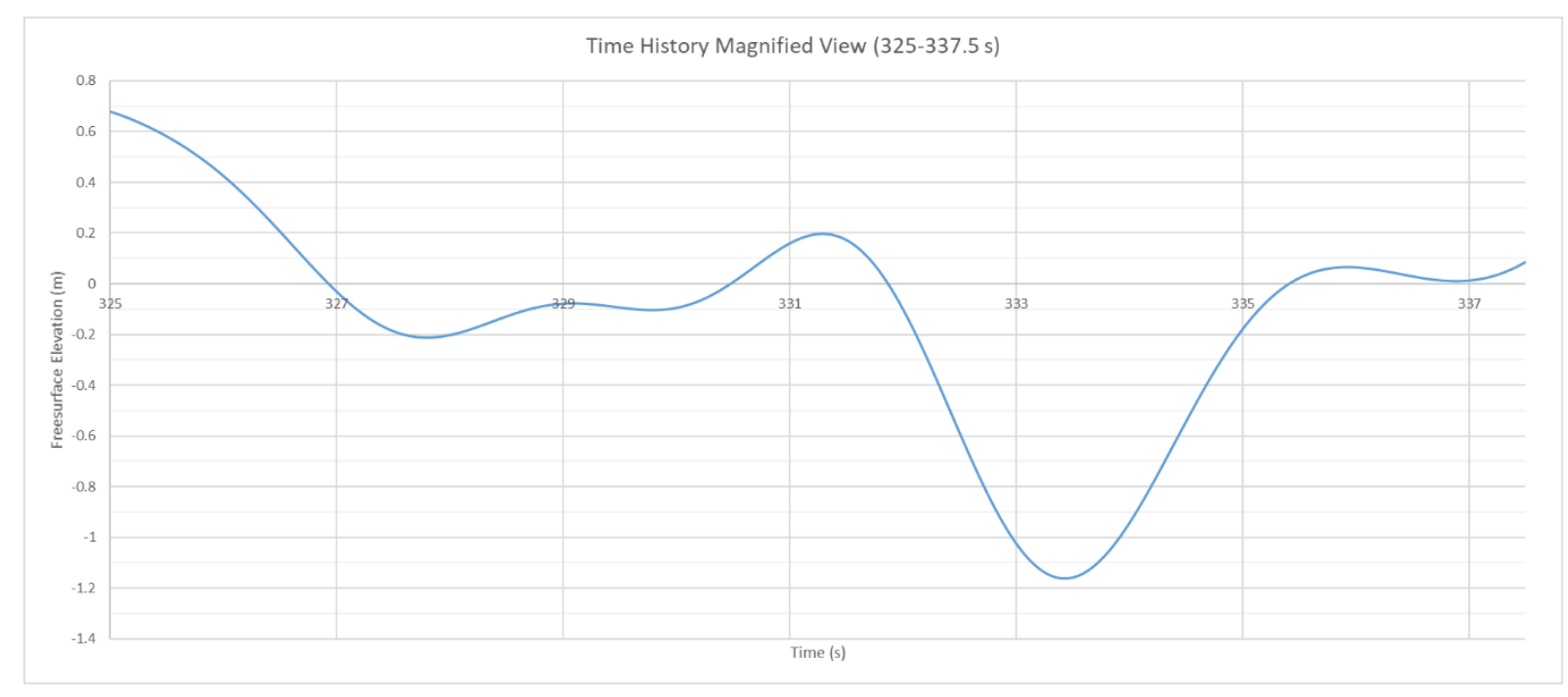

Figure 8 Magnified view of wave elevation between 325-337.5 seconds to view the smallest observed wave

As shown in the validation and verification study above, the mesh scheme developed in this paper achieved an error of less than $1 \%$. This error margin is significantly below the estimates laid out above. This is due to two main reasons. Firstly, the mesh scheme and time step used were very close to the estimated optimal values, but predominately the second reason in the selection of simulation time. As the simulations in the V\&V study used the test mode within the JONSWAP spectrum, a simulation time was selected to match the 300 encountered waves but also ensured the observed Hs matches within $0.5 \%$ of the desired value. This is significantly below the theoretical maximum potential error limit of $-6.05 \%$ and $9.62 \%$ respectively, as estimated based upon the data from Figure 15. Further to this, the initial 300 seconds of the wave elevation were analysed to compare with the other sea states due to their reduced simulation time. The Hs error seen for 300 seconds increased from $0.5 \%$ to $2.43 \%$. This value fits the observed pattern of error deviation seen in Figure 15 when compared with the error value found for 1500 seconds. As seen from Figure 15, the theoretical maximum potential error increases as the simulation time decreases, this increases is around 4.75 times from 1500 to 300 seconds. Based upon this observation, an estimated error of $2.375 \%$ at 1500 seconds, which is marginally lower than actual observed error. These values are both around 10 times lower than the calculated theoretical maximum limits from Figure 15 of around $30 \%$. This is likely due to relatively consistent wave elevations over the 300 second period, as can be seen in Figure 10. 


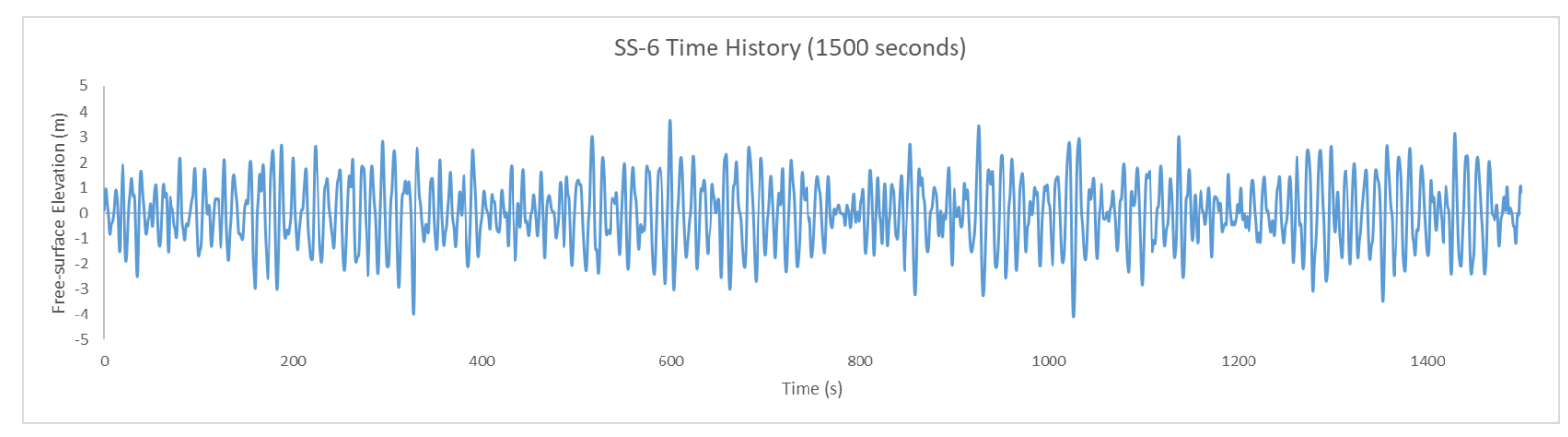

Figure 9 CFD free surface elevation for SS-6 over 1500 seconds

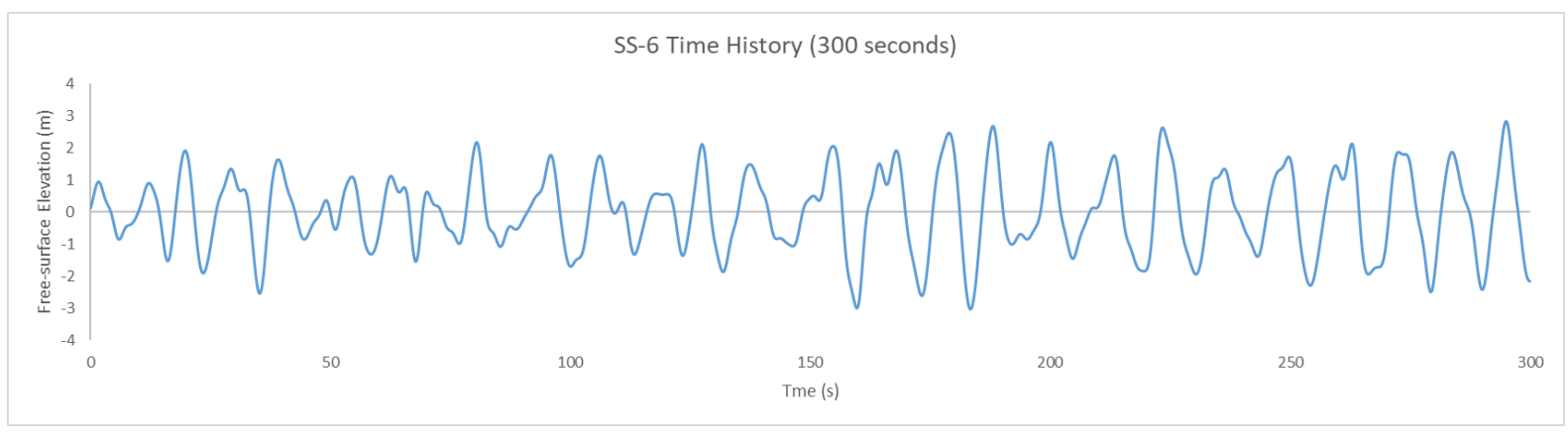

Figure 10 CFD free surface elevation for initial 300 seconds for SS-6

Due to the size and complexity of the simulation, the only practical way to run the simulation was through the use of an High Performance Computer (HPC, Archie WEST). The simulation took in excess of to $675,000 \mathrm{CPU}$ hours for 1500 second simulation time. This would have been reduced to 530,000 CPU hours due to the final period of the simulation running on a hardware updated HPC. This length was due to the simulation needing to model both small and large waves over the full range of Sea State 6 limits. This highlights the major drawback to such a simulation for practical applications at the current point in time.

\subsection{Sea State 5}

In addition to SS-6 (sea state), both SS-5 \& SS-4 were also investigated to analyse the accuracy of the methodology and the running time for various sea states. For SS-5 the calculated mesh variables are shown in Table 8, along with the prospective errors. As can be seen, the minimum cell size is much smaller than with respect to the relative significant wave heights. In addition to the reduced cell size, the number of cells in the $\mathrm{Z}$ height for the first refinement region should remain constant, however when the mesh has been generated the smallest $\mathrm{Z}$ height that could be modelled was $18 \%$ larger than desired. Leading to a cell height of $0.0117(\mathrm{~m})$, this in turn, caused a reduction in the number of cells per minimum wave height from 16 to 13 . This still remained within the limits advised by Star CCM+, however this meant that a much smaller margin of lower wave heights can be captured in the mesh. This was seen in the form of the smallest stable wave being only $0.1037(\mathrm{~m})$, a reduction of around $6 \%$ from the minimum modelled height of $0.1098(\mathrm{~m})$. This is a significant reduction compared with the $30 \%$ seen in SS-6. 
Table 8 Calculated cell heights and distributions for a Sea State 5 simulation

\begin{tabular}{|c|r|}
\hline Sea State & 5 \\
Hs & $3.25 \mathrm{~m}$ \\
Tp & $9.7 \mathrm{~s}$ \\
Spectrum & JONSWAP \\
\hline
\end{tabular}

\begin{tabular}{|l|r|}
\hline Av Wave Steepness & 0.0221 \\
\hline
\end{tabular}

\begin{tabular}{|c|r|rl|}
\hline $\begin{array}{c}\text { Max Z height in +ve \& -ve } \\
\text { Min Wave Height }\end{array}$ & $\begin{array}{r}1.3089 \mathrm{~m} \\
0.11 \mathrm{~m}\end{array}$ & \\
& FrSf_2 & FrSf_3 \\
\hline Free Surface Refinement Region & FrSf_1 & & \\
\hline No of Cells per Wave Height & $0.0097 \mathrm{~m}$ & $0.0194 \mathrm{~m}$ & $0.0388 \mathrm{~m}$ \\
\hline Z cell Height & & & \\
\hline
\end{tabular}

\begin{tabular}{|c|c|c|c|}
\hline Min Wave length & $4.9626 \mathrm{~m}$ & & \\
\hline No of Cells per $L$ & 60 & 4 & 4 \\
\hline X Cell Length & $0.0830 \mathrm{~m}$ & $0.166 \mathrm{~m}$ & $0.332 \mathrm{~m}$ \\
\hline Y Cell Length & $0.0830 \mathrm{~m}$ & & \\
\hline
\end{tabular}

Although the over cells sizes have been reduced, thus causing an increase in potential overall cell number, the overall cell number is slightly lower than SS-6 due to the reduction in overall domain size caused by the decreased maximum wave length. This trend is further seen in SS-4, suggesting smaller sea states become more computationally efficient. This however, does not make the simulations quicker at a similar rate, as the time steps required become smaller due to the reduced cell size. It is noted that although there is limited equalling effect of reduced cell numbers and time step size, a SS-5 simulation ran around $10 \%$ quicker than an equivalent SS-6 simulation.

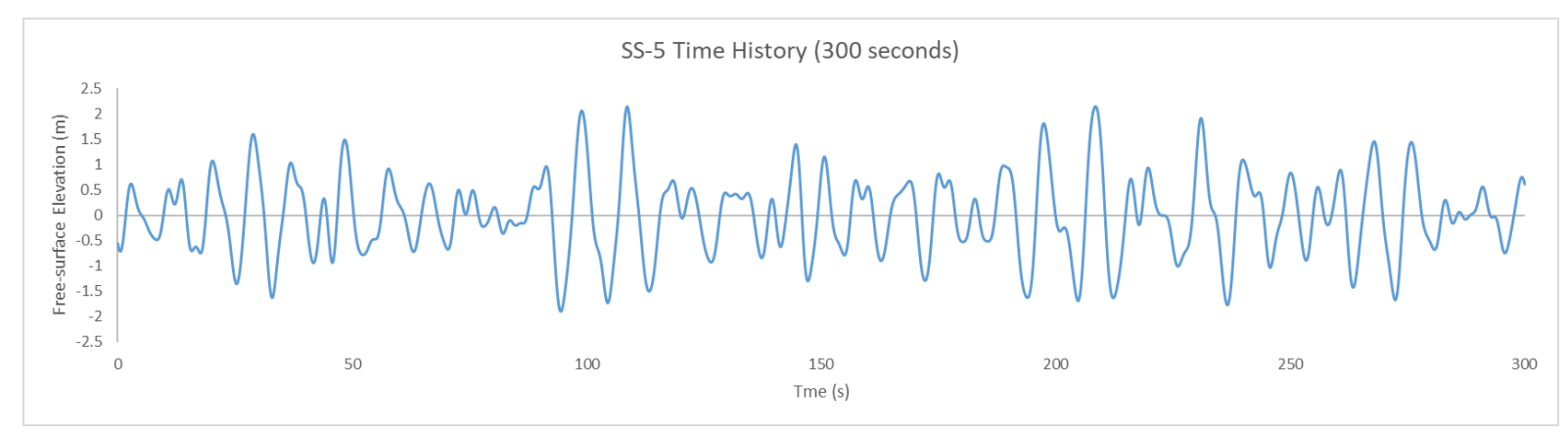

Figure 11 CFD free surface elevation for initial 300 seconds for SS-5

The calculated Hs derived from the simulated waves matched the desired Hs within 5\%, which is still significantly below the error cone discussed on section 2.1 , which highlighted a potential maximum 
observer error of $27 \%$ for a simulated time of 300 seconds. The observed time history is shown in Figure 11. This also highlights the increase in observed waves within the simulated time compared with SS-6.

Two reasons led to the reduced running time of 300 seconds, the first was practical hardware limitations and the second was to apply the simulations in a more practical simulated approach. Although practical applications may reduce the simulation even further, as mentioned in section 2.8, 300 seconds was chosen as the time allowed a large variety of waves to travel through the domain and thus produce a small but accurate snap shot of the irregular sea. The overall simulation required around $1.25 \mathrm{e} 5 \mathrm{CPU}$ hours to run. This further highlights the hard ware and software requirements of such a simulation.

\subsection{Sea State 4}

As mentioned above multiple sea states have been used to test the methodology put forward in this paper. However SS-4 was also testing the greater practicality of the methodology. As it is becoming a much greater interest to both research's and industry to test simulation in real world conditions, therefore SS-4 was selected as it represents a common extreme worked in by offshore industries.

The estimated cell sizes and time steps are shown in Table 9. Based upon these values, overall 20 million cells were used. A reduction of around 5 million cells compared with SS-5. As with the previous simulations, the generated cells varied slightly compared with the calculated values. The variation increased significantly for SS-4 in cell height, with a deviation of $20 \%$. This caused the cells per minimum wave height to reach the minimum limit of 12 , thus leading to no further reductions in simulated waves.

The simulated Hs for the 300 periods came to 1.724 (m) that equated to a deviation of $9.1 \%$ from the desired Hs of $1.88 \mathrm{~m}$. The simulated wave elevation for SS-4 is shown in Figure 12. This error remained within the maximum potential limits of around 25\%, as estimated in Figure 17 in Appendix iv.

This cell size increase was due to the meshing scheme within CFD code, as it failed to accurately match the desired inputs and aspect ratios, thus leading to a compromised mesh that did not allow for any smaller waves than the calculated minimum to be simulated. This also matched the previously mentioned trend of smaller wave height simulations having a much lower ability to model smaller waves than the calculated minimum wave height. This becomes a significant problem, as the cell numbers within a small simulation can become too large to be considered advantageous or practical. 
Table 9 Calculated cell heights and distributions for a Sea State 4 simulation

\begin{tabular}{|c|r|}
\hline Sea State & 4 \\
$\mathrm{Hs}$ & $1.88 \mathrm{~m}$ \\
$\mathrm{Tp}$ & $8.8 \mathrm{~s}$ \\
Spectrum & JONSWAP \\
\hline
\end{tabular}

\begin{tabular}{|l|l|}
\hline Av Wave Steepness & 0.0155 \\
\hline
\end{tabular}

\begin{tabular}{|c|r|rl|}
\hline $\begin{array}{c}\text { Max Z height in +ve \& -ve } \\
\text { Min Wave Height }\end{array}$ & \multicolumn{1}{|c|}{$\begin{array}{l}0.7684 \mathrm{~m} \\
0.0645 \mathrm{~m}\end{array}$} & & \\
\hline Free Surface Refinement Region & FrSf_1 & FrSf_2 & FrSf_3 \\
\hline No of Cells per Wave Height & 16 & & \\
\hline Z cell Height & $0.0054 \mathrm{~m}$ & $0.0107 \mathrm{~m}$ & $0.0215 \mathrm{~m}$ \\
\hline
\end{tabular}

\begin{tabular}{|c|r|rr|}
\hline Min Wave length & $4.1457 \mathrm{~m}$ & & \\
\cline { 2 - 2 } No of Cells per L & 60 & 4 & 4 \\
X Cell Length & $0.0691 \mathrm{~m}$ & $0.138 \mathrm{~m}$ & $0.276 \mathrm{~m}$ \\
\cline { 2 - 2 }
\end{tabular}

\begin{tabular}{|l|l|}
\hline Y Cell Length & $0.0691 \mathrm{~m}$ \\
\hline
\end{tabular}

Minimal tweaking of the time step was required, in this case the time step was increased slightly from the calculated value without causing instabilities within the simulation. The increased time step led to further reduction in overall simulation time and CPU usage, with a $17 \%$ reduction in CPU hours used compared with a $20 \%$ reduction in cell numbers. The simulation used $1.1 \mathrm{e} 5 \mathrm{CPU}$ hours to reach 300 seconds. However, due to the smaller waves, a greater number of waves entered the domain compared with the previous simulations, this can be seen clearly in Figure 13, it is, therefore, possible to reduce the overall run time to account for the increased number of waves seen per minute.

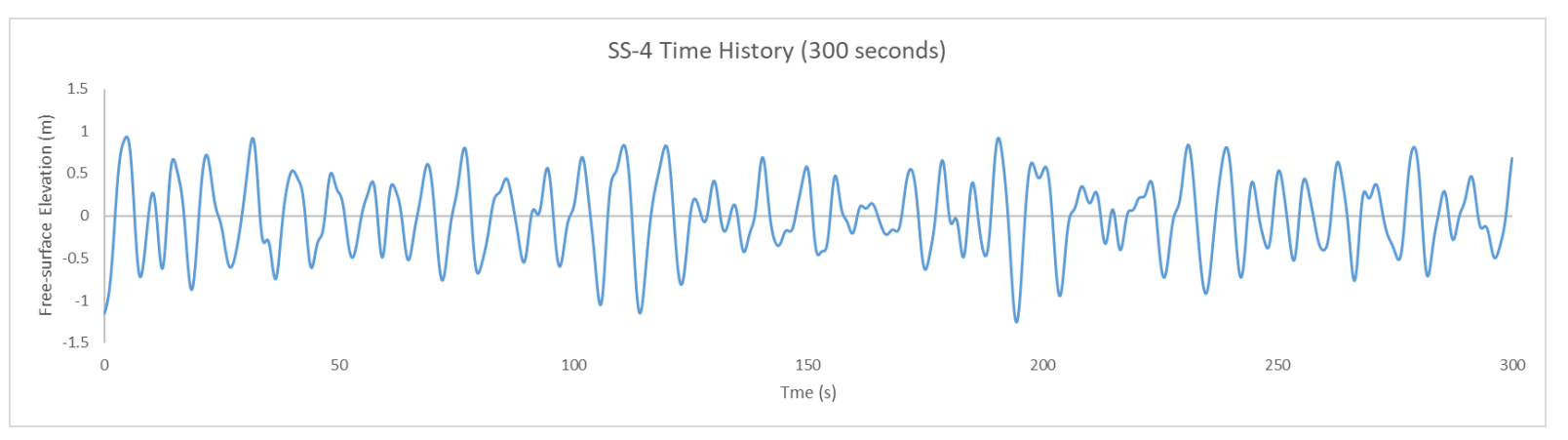

Figure 12 CFD free surface elevation for initial 300 seconds for SS-4 


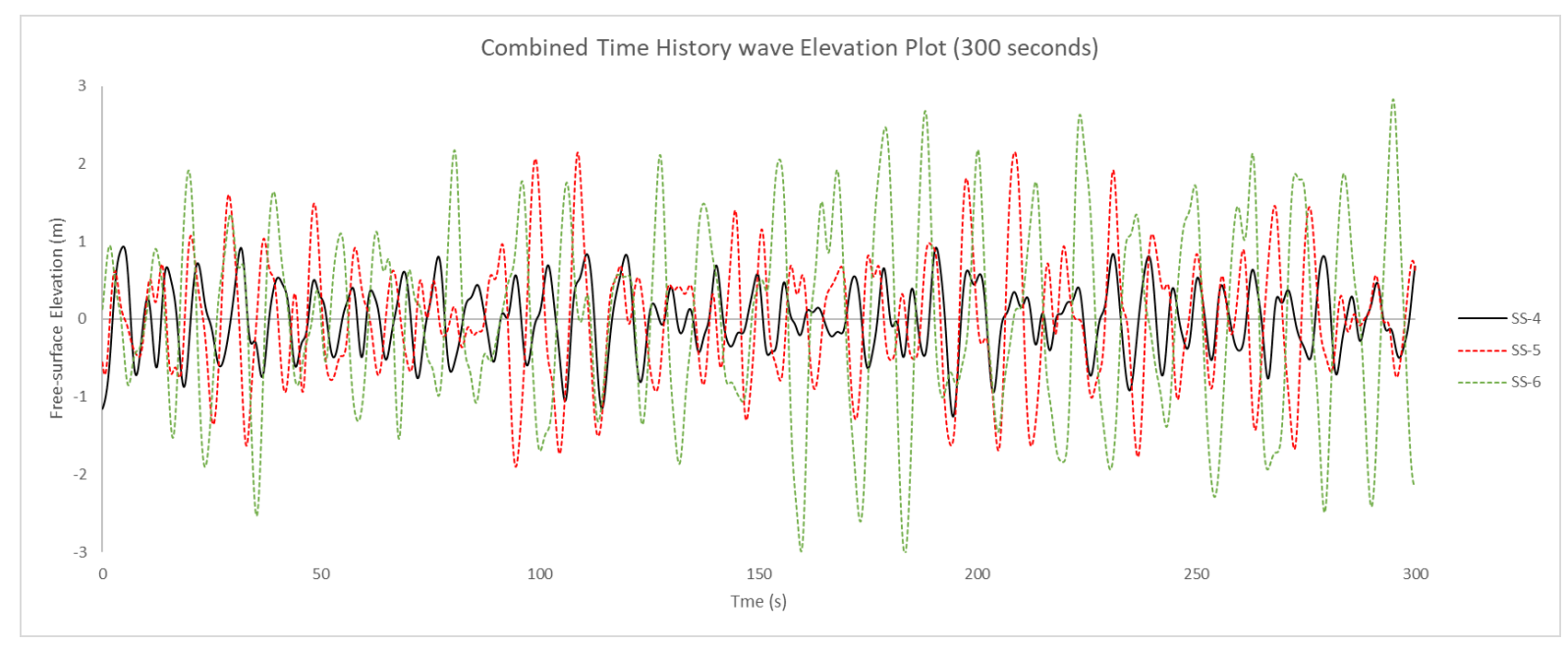

Figure 13 Comparison of CFD Time histories for simulated sea states

As can be seen from the discussions above, all the simulations fell well within the theoretical maximum error margins estimated through the in-house code and shown in Figure 3, Figure 15 and Figure 17 in appendix iv. These graphs also show that as the Hs decreases, the theoretical error margin for smaller run times also decreases due to the reduced average wave period and thus an increase in a number of observed waves, this is shown clearly in Figure 19. 


\subsection{Concluding Remarks}

This section will provide a final overview of the work conducted above, along with the key factors relating to the best practices for simulating unknown irregular seas.

Through the study looking at various sea states, multiple points can be drawn for both the results obtained, along with the overall methodology. Each simulation achieved a level of accuracy that satisfies the simulation time error, along with the additional mesh errors. This shows that the methodology of decomposing an irregular sea in to key factors guarantees the ability to capture the desired sea state with respect to accuracy levels needed required that simulation. As noted above, these accuracy levels vary greatly with respect to the mesh density, estimated time steps, and overall simulation. Two examples of varying accuracy levels may be as follows:

1. A small wind and current device located in a minor harbour, such that all wave heights are vital to the operation of the system, thus a high mesh count and accuracy level is critical to ensuring valid simulation results

2. An ocean-going vessel, such as a large containership or anchor handling tug, experiencing a large sea state and variable wind conditions, to investigate vessel motions and performance limitations. Such a simulation may require a large full domain to allow variable wave directions. Thus the requirement for the smallest waves may be ignored, to a certain degree, due to the large moments and forces required to instigate motions, and thus helping to reduce cell numbers.

These examples highlight both the importance of the engineers understanding of the subject being simulated, along with an initial methodology to work off and vary based upon the simulation.

It can be seen from the results discussed throughout section 4, that the current values used and proposed in the creation of the simulations may be over refining the mesh density. This, in turn, causes the simulation to be slower than the required for the final desired accuracy level, this is most seen when comparing SS-6 and SS-4 simulations. Both achieve the desired accuracy level, with SS-6 being closer to the desired aim, while SS-4 Hs is only slightly less accurate but with a significant reduction in cell numbers comparatively. It is therefore advised that the initial mesh should aim to be over refined and then to coarsen the mesh. An initialisation of the free-surface within the domain will further help to identify if the mesh is over refined or at the correct level for the simulation.

The simulations maintained the desired accuracy, with the minimum wave captured travelling through the domain being below the desired smallest wave height according to the mesh coarsening. Therefore, although the mesh was coarsened, the relative error from the coarsening was reduced. Further to this, the methodology has proven accurate in modelling the desired time history. However such simulations are very dependent upon the simulated time and the respective time history error. In order to effectively 
analyse the time history error within a simulation, the simulated free-surface should have a Fourier analysis conducted to compare with the desired wave spectrum. This ensures that an observed error may be due to either the time history error margin or inaccuracies within the simulation. The second factor will require a reworking of the simulation.

The ability to accurately model such conditions will become invaluable when attempting to simulate ever more realistic ocean conditions for fatigue and motion simulations for offshore or coastal engineering projects. In addition to increasing the accuracy for estimating complex drag problems for both marine vehicles and structures.

As discussed and highlighted above, irregular sea simulations require a significant amount of computational power and time. It is therefore vital that any research or application within the industry conduct a preliminary statistical analysis of the desired sea state to be modelled. Not only does this provide a clear benchmark to later validate against, but provides the initial steps in defining the accuracy level desired for the specified work as seen in the case study section of this study.

From post-processing analysis of the modelled time histories, the key values relating to the minimum desired wave height, the maximum height for the fine refinement region, and the statistical maximum wave height can be drawn. These values can be used, as described in Section 2, to define the refinement volumes, along with the key cell length required to maintain the specified Hs. These can be calculated by using the equations defined by (Arena et al. 2010) to determine the wave lengths, based upon the wave height and in turn the wave periods. These equations can be seen in section 2.1 , defined as equations (8) \& (9).

To further ensure simulation stability, the maximum aspect ratio for the cells within the free-surface refinement regions should not exceed 15 with respect to $X \& Z$. It is possible to increase the cell length in the $\mathrm{Y}$ direction in the far field regions to reduce the overall cell numbers used in the simulation.

Upon the mesh reaching the desired accuracy and refinement level, the respective time step must be defined. Depending upon the simulation, the time step may be defined using the equations listed above in section $3.7,(13),(14),(15) \&(16)$.

The overall simulation time must be selected, and as prescribed above a minimum of 100 waves should be used only when used with a Discrete Fourier Transform (DFT), as describeded by Mousaviraad (2010), and all other simulations should look to encounter a minimum of 300 waves or a simulation time of 1.5 hours.

As highlighted from Section 4, the numerical setup defined in Section 2 assumes an optimum setup and therefore may be tweaked to reduce cell numbers and running time. This, however, should be done with care to remain as close to the described best practices above as possible. 
It can be concluded that such simulations come with a significant requirement for computational power and time. It can also be drawn that conducting such simulations in full scale can elevate any potential scaling issues when modelling the waves, as the mesh number will remain constant for the free surface region independent of model or full scale.

Further to this, this study has provided an initial baseline investigation for an alternate method other than the superposition methods currently in the literature. As highlighted above, this method aimed at improving the accuracy of modelling non-linear irregular seas at a greater accuracy than the commonly used superposition methods. The primary limitation of current superposition methods is the number and range of waves being modelled by the user, whereas the method developed above uses spectrum based data and thus has a significantly larger range of waves used to model the sea state. Due to the increased range, the wave interactions are modelled more accurately, and in turn the complex non-linear effects. Although the method described in this paper enables a more accurate simulation of non-linear waves, it is significantly more computationally heavy than the superposition method. This is most noticeable of short run time simulations, as both the simulation requires more computational power and there is no guarantee of achieving the desired Hs being modelled for compared with the superposition methods. However, for longer run time simulations the ability to model more complex wave interactions and nonlinear effects the presented method becomes more appealing. Due to the need to increase the number of user defined waves in the superposition method, the advantages become negated while still producing limitations to the overall complexity of the sea state.

This study has provided the preliminary foundations for future CFD work relating to non-linear unknown irregular sea simulations to be worked upon. Such research should investigate the effects of varying wind conditions and the corresponding effect on the free surface, thus bringing CFD closer to full modelling of real-world conditions.

Further research should be conducted looking in to marine structure interactions and the effects on the described methodology by utilising this full domain rather than half domains with symmetry planes, with varying wave headings and the respective effect on added resistance and motions. It should also be considered in applying propellers and other appendages to investigate the effects of such sea states on these factors and how to improve complete vessel performance in irregular seas.

Further research should aim to investigate how optimisation using irregular seas can be achieved, and how this can be applied within the industry to reduce the GHG emissions of the marine industry. The research should focus on utilising transfer functions to validate vessel performance in such a method that the second order characteristics of added resistance are satisfied. 


\section{Acknowledgements}

The study presented in this paper was carried out as part of the research project: 'An automated optimisation of a fully parametric vessel for real world conditions' funded by EPSRC Grant no. EP/N509760/1 and the University of Strathclyde. Results were obtained using the ARCHIE-WeSt High Performance Computer (www.archie-west.ac.uk) based at the University of Strathclyde. It should also be noted that there is no data underpinning this publication. 


\section{Appendix i}

Table 10 Mesh estimation scheme, sea state 6

\begin{tabular}{|cr|}
\hline LOA & \\
\hline Domain $X$ & $100 \mathrm{~m}$ \\
Domain $\mathrm{Y}$ & $75 \mathrm{~m}$ \\
Domain $\mathrm{Z}$ & $75 \mathrm{~m}$ \\
\hline
\end{tabular}

\begin{tabular}{|c|r|}
\hline Sea State & 6 \\
$\mathrm{Hs}$ & $5 \mathrm{~m}$ \\
$\mathrm{Tp}$ & $12.4 \mathrm{~s}$ \\
\hline Spectrum & JONSWAP \\
\hline
\end{tabular}

\begin{tabular}{|c|c|}
\hline Av Wv & \\
Steepness & 0.0208275 \\
AWS & $\mathrm{H} / \mathrm{L}$ \\
\hline
\end{tabular}

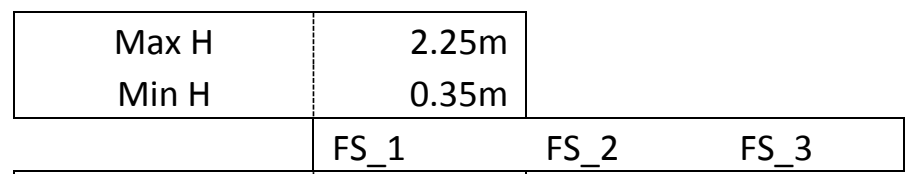

\begin{tabular}{|c|r|rr|}
\hline No of Cells per $\mathrm{H}$ & 16 & & \\
\hline Z cell Height & $0.021875 \mathrm{~m}$ & $0.04375 \mathrm{~m}$ & $0.0875 \mathrm{~m}$ \\
\cline { 2 - 4 } & & $0.7 \mathrm{~m}$ & $1.4 \mathrm{~m}$ \\
\cline { 2 - 4 } & 16 & $2.95 \mathrm{~m}$ & $4.35 \mathrm{~m}$ \\
\hline
\end{tabular}

\begin{tabular}{|c|r|rr|}
\hline Min L & $16.805 \mathrm{~m}$ & & \\
No of Cells per L & 60 & 4 & 4 \\
X cell & $0.2801 \mathrm{~m}$ & $0.5602 \mathrm{~m}$ & $1.1204 \mathrm{~m}$ \\
\hline Delta Volume & & $2.2406 \mathrm{~m}$ & $4.4813 \mathrm{~m}$ \\
\cline { 2 - 3 } & &
\end{tabular}

\begin{tabular}{|l|l|}
\hline Y cell & $0.2801 \mathrm{~m}$ \\
\hline
\end{tabular}

\begin{tabular}{|l|r|}
\hline Volume Ratio X & 0.85 \\
Volume Ratio Y & 1 \\
Volume Ratio Z & 0.8 \\
\hline
\end{tabular}

\begin{tabular}{|l|l|}
\hline Cell Volume FS1 & $0.00612 \mathrm{~m}^{3}$ \\
Cell Volume FS2 & $0.00686 \mathrm{~m}^{3}$ \\
Cell Volume FS3 & $0.02745 \mathrm{~m}^{3}$ \\
\hline
\end{tabular}

\begin{tabular}{|l|r|r|r|}
\hline volumes & \multicolumn{3}{|c|}{} \\
\hline $\mathrm{x}$ & $85 \mathrm{~m}$ & $87.240 \mathrm{~m}$ & $91.721 \mathrm{~m}$ \\
$\mathrm{y}$ & $75 \mathrm{~m}$ & $75 \mathrm{~m}$ & $75 \mathrm{~m}$ \\
$\mathrm{z}$ & $2.25 \mathrm{~m}$ & $3.65 \mathrm{~m}$ & $5.05 \mathrm{~m}$ \\
volume & $14343 \mathrm{~m}^{3}$ & $23882 \mathrm{~m}^{3}$ & $34740 \mathrm{~m}^{3}$ \\
\hline
\end{tabular}




\begin{tabular}{|l|r|r|} 
Delta Volume & $9539 \mathrm{~m}^{3}$ & $20397 \mathrm{~m}^{3}$ \\
\hline
\end{tabular}

\begin{tabular}{|l|l|l|l|}
\hline No Z & 102.857 & \\
No X & 303.486 & \\
No Y & 267.782 & & Est Free Srf Cell Number \\
\hline
\end{tabular}


Appendix ii

Raw JONSWAP spectrum time history, post-processing analysis and mesh value calculations.

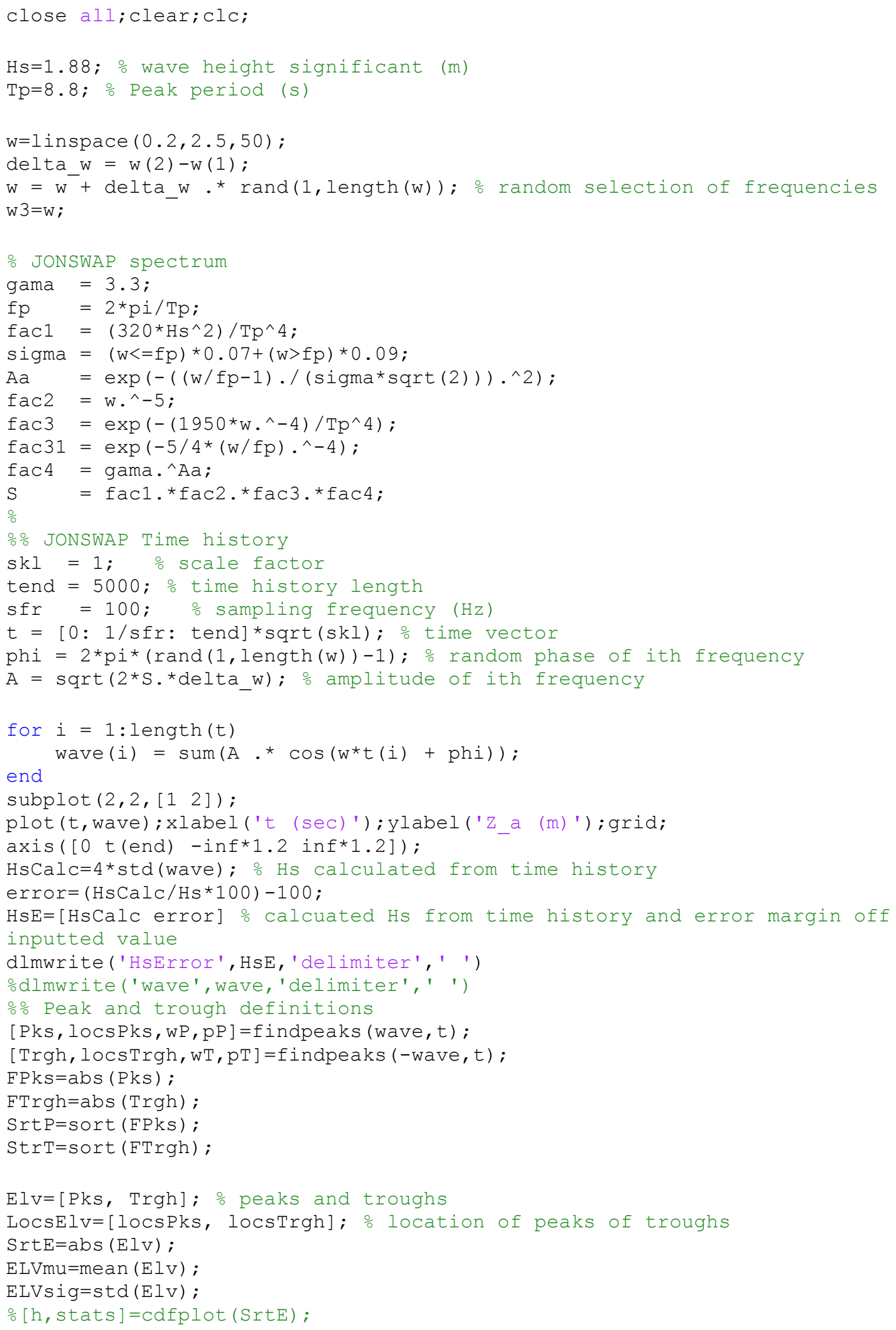




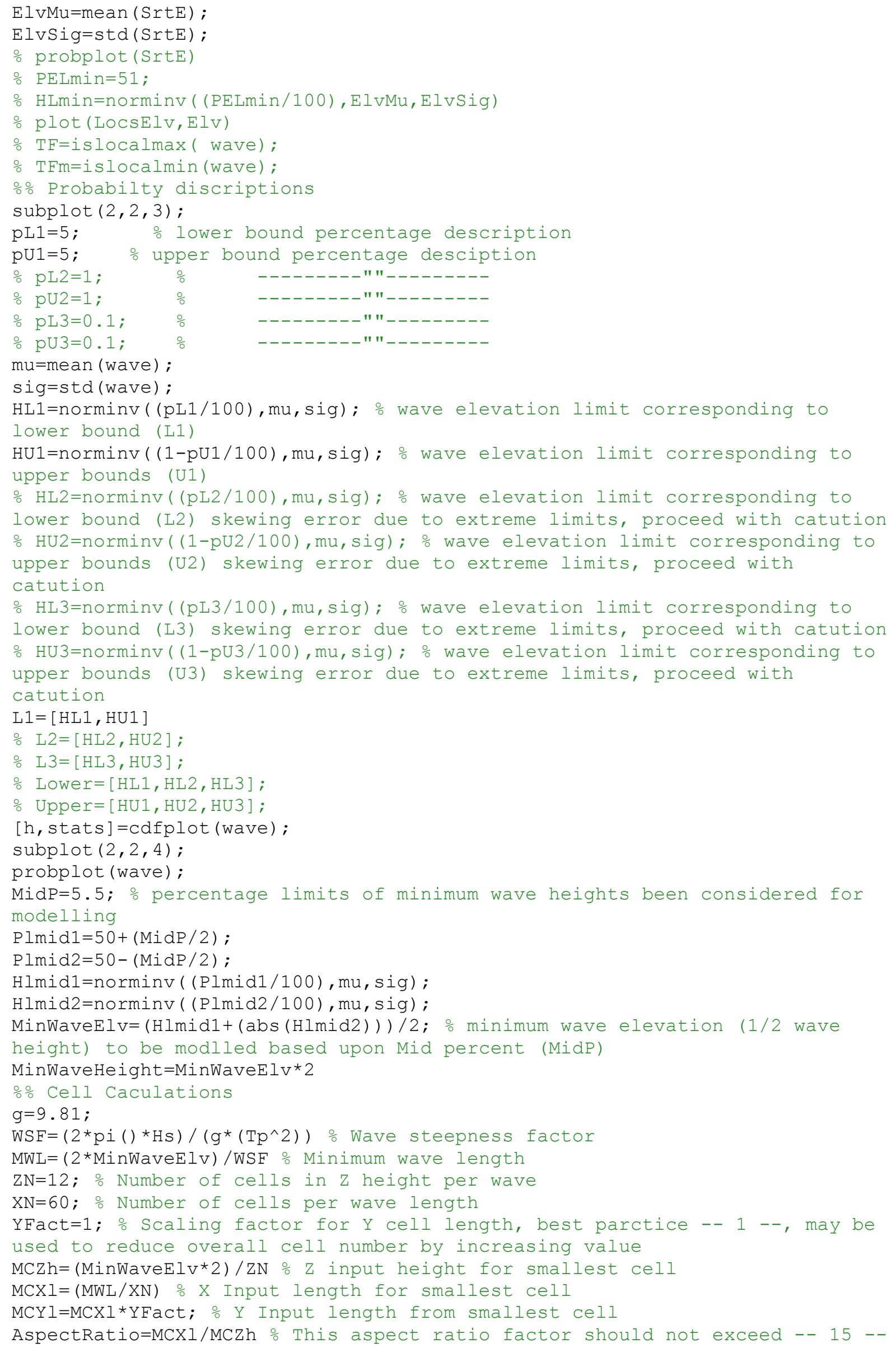


FS1CN=ceil( (abs (HL1)+HU1)/MCZh) \% estimated number of cells in intial free surface region 


\section{Appendix iii}

\section{Worked example of MATLAB code with key inputted values}

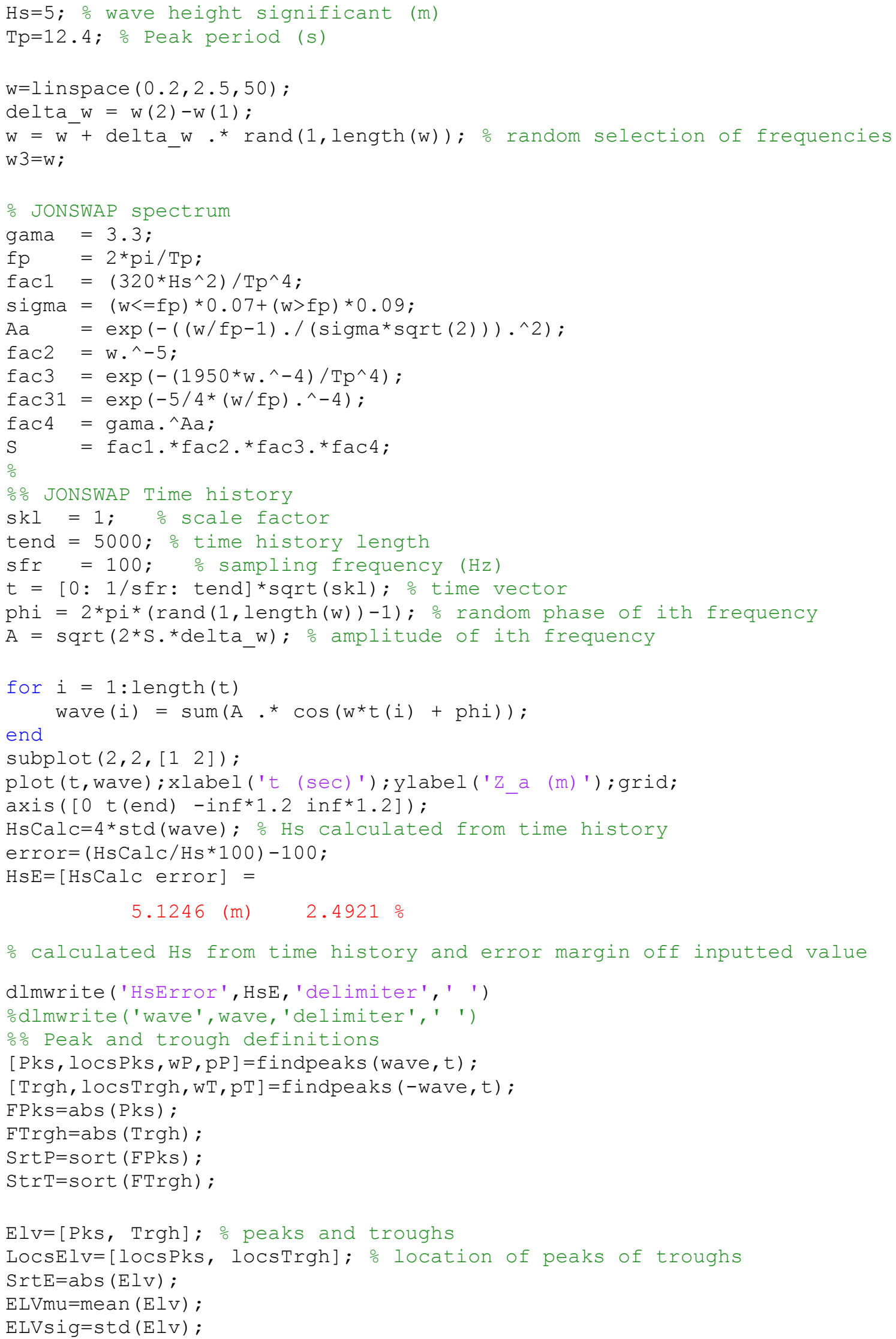




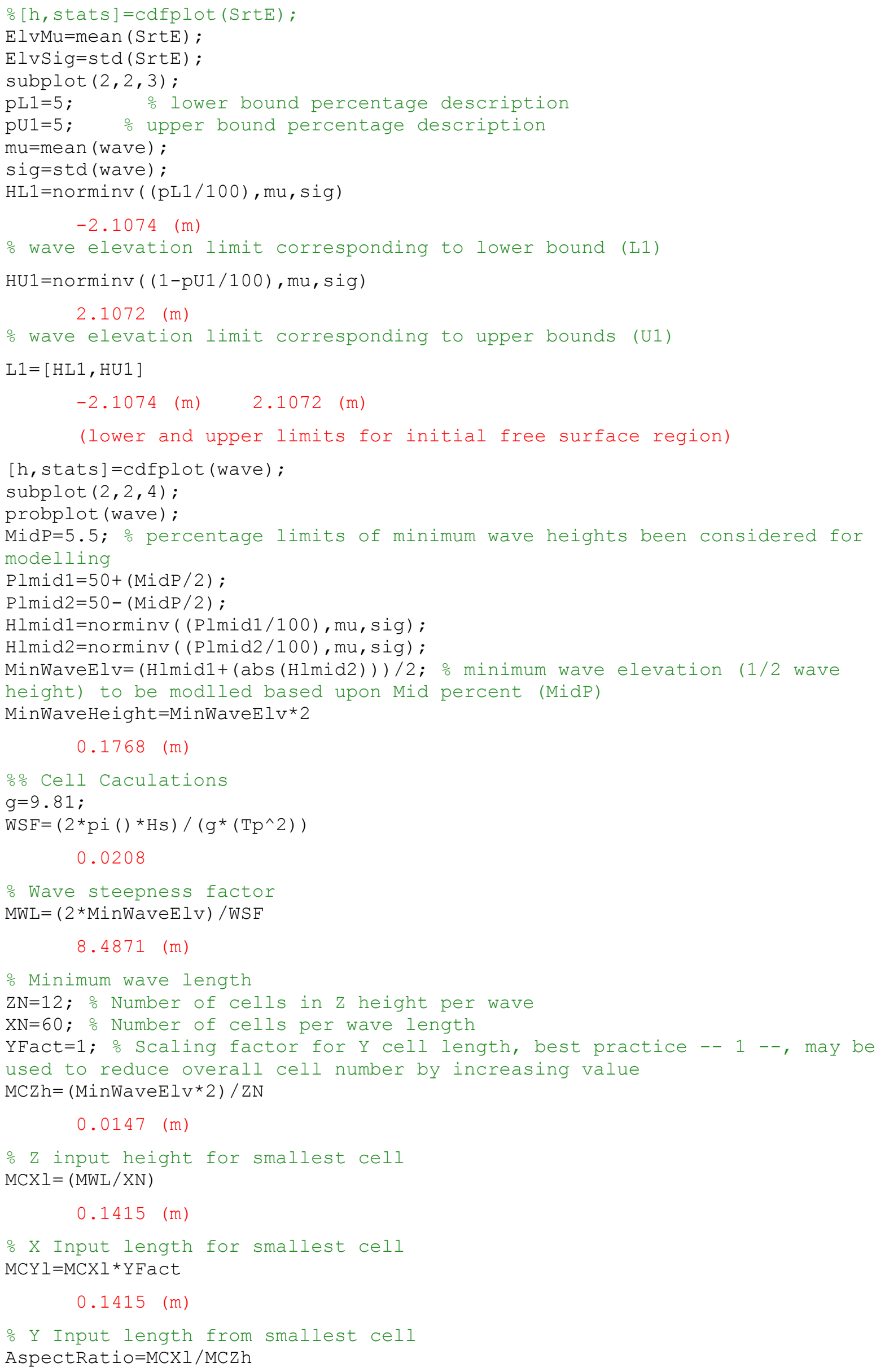


$9.6027<15$ therefore good size limits

o This aspect ratio factor should not exceed -- 15 --

FS1CN=ceil ( (abs (HL1) +HU1)/MCZh)

287

\% estimated number of cells in initial free surface region
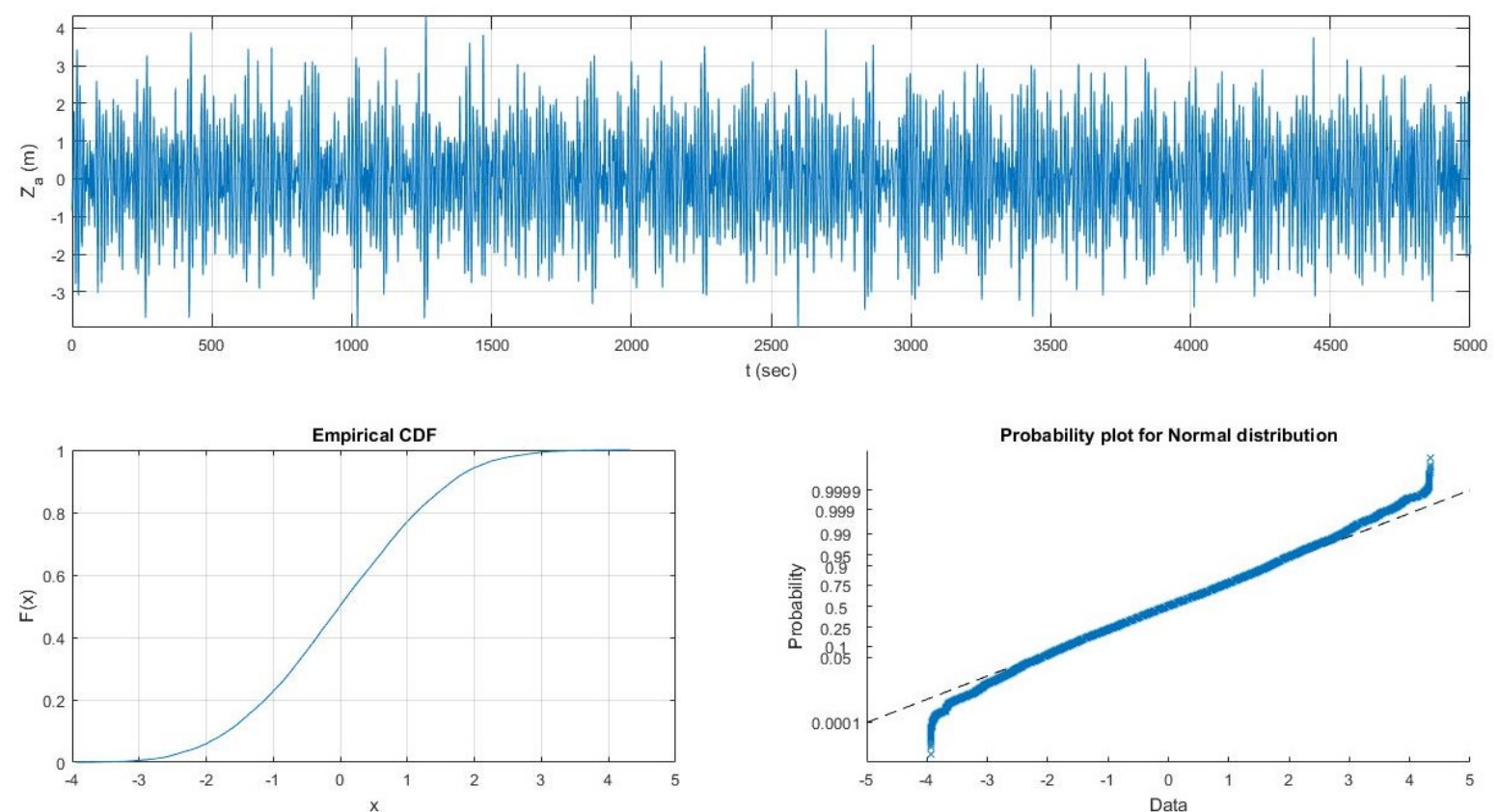

Figure 14 Graphical output from JONSWAP Matlab code for Hs $=5(\mathrm{~m}), \mathrm{Tp}=12.4(\mathrm{~s}) \& \mathrm{~T}=5000(\mathrm{~s})$ 


\section{Appendix iv}

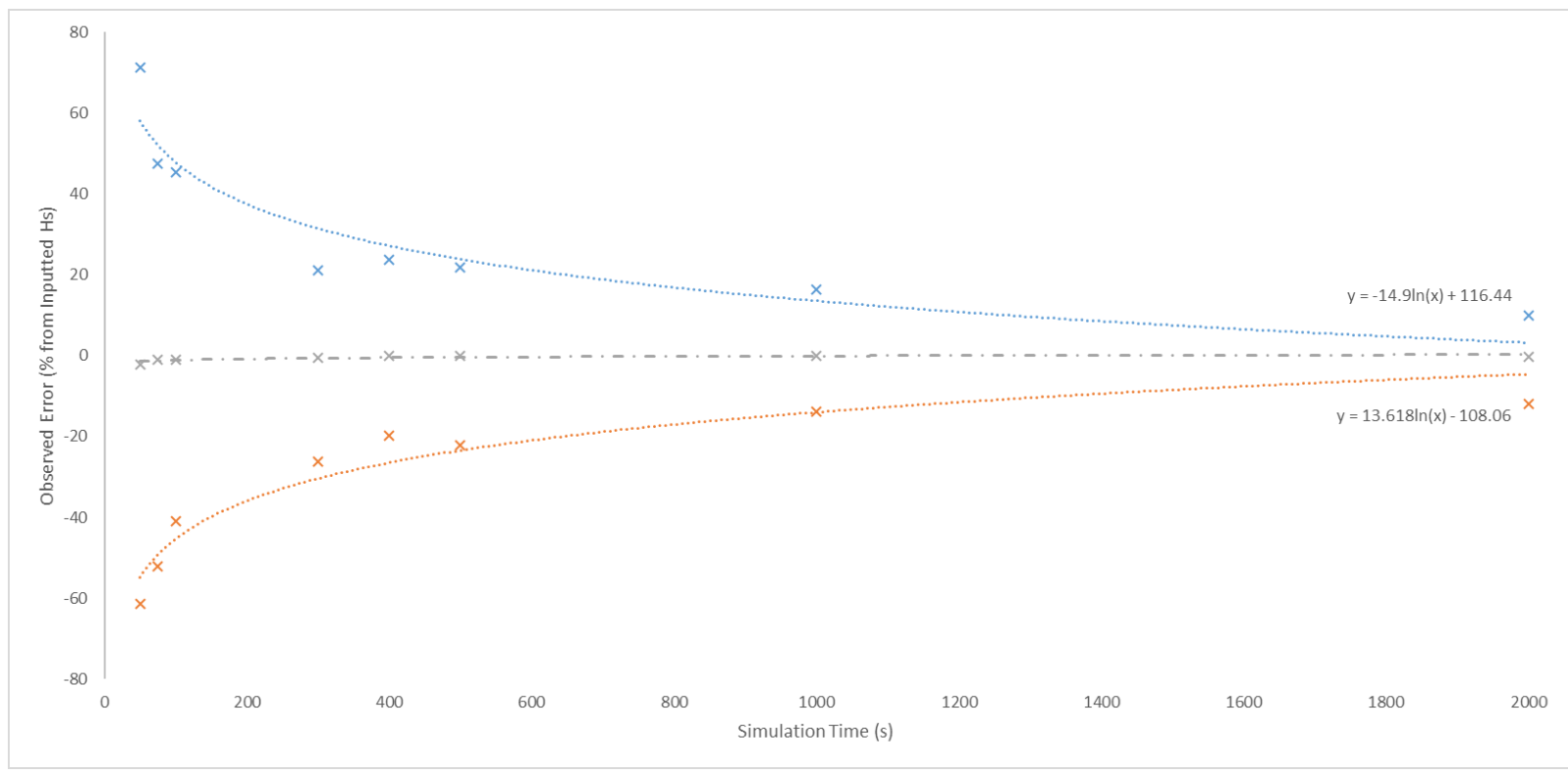

Figure 15 SS-6 Theoretical error margins with respect to MATLAB simulation time

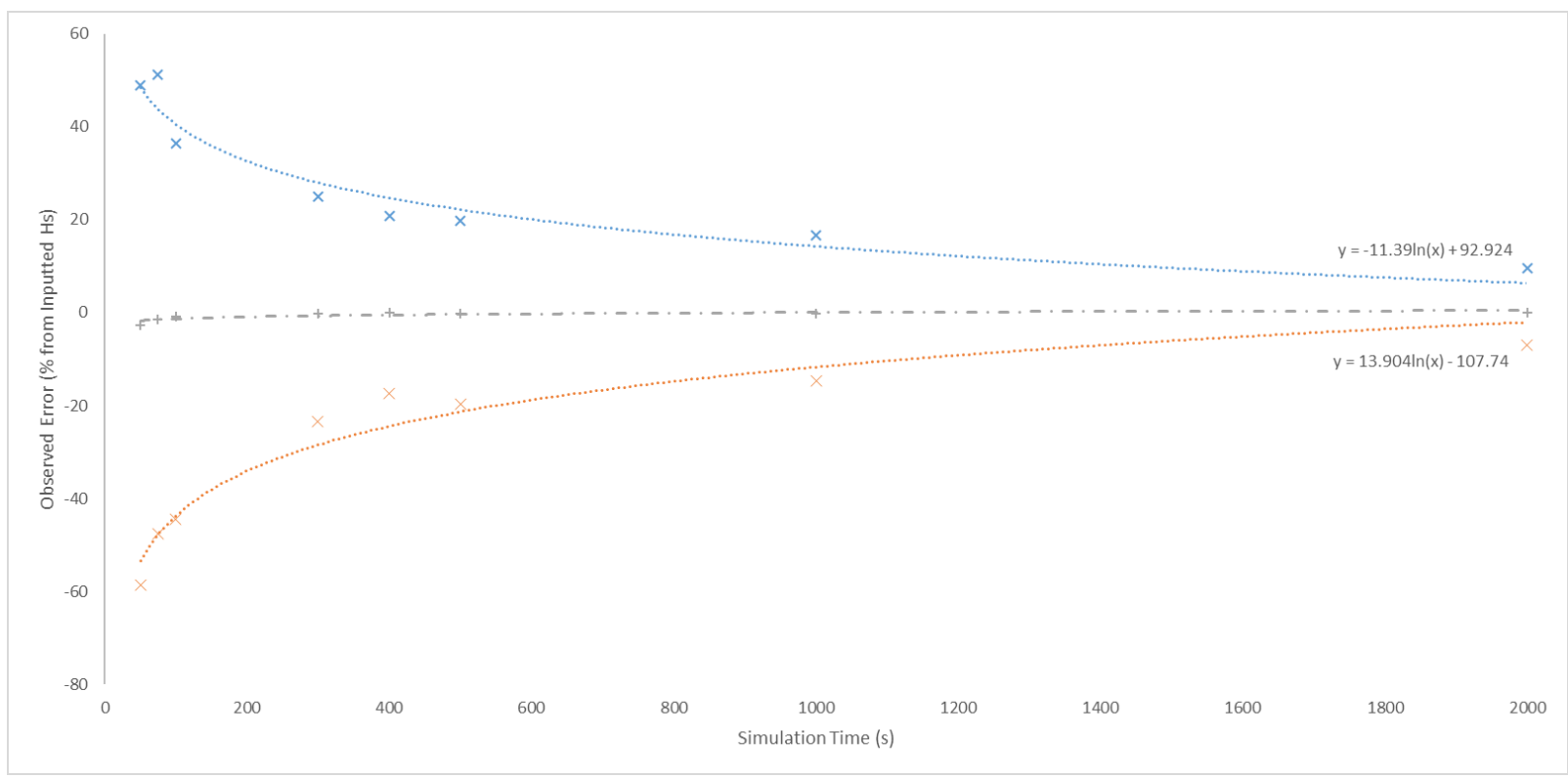

Figure 16 SS-5 Theoretical error margins with respect to MATLAB simulation time 


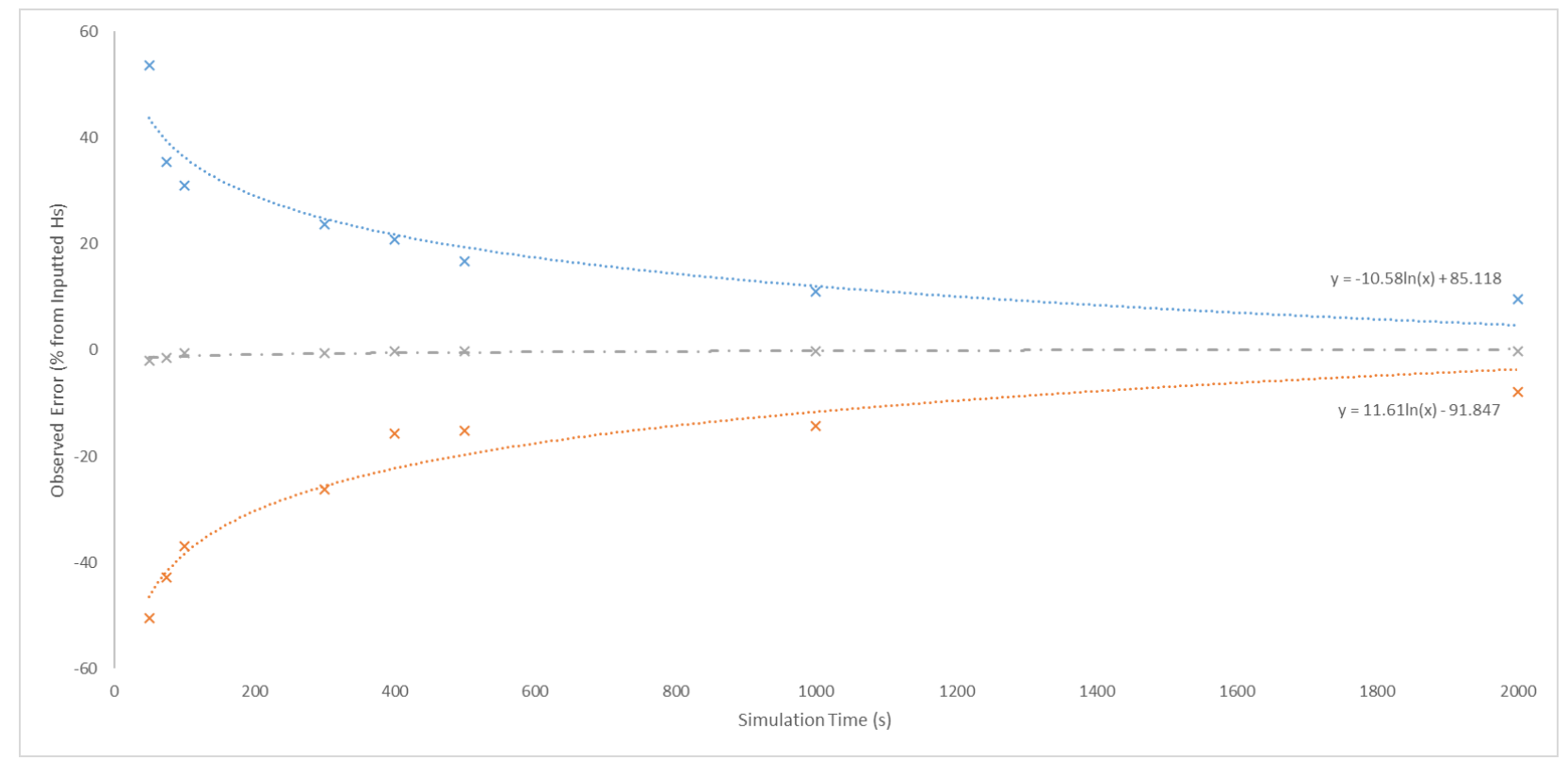

Figure 17 SS-4 Theoretical error margins with respect to MATLAB simulation time

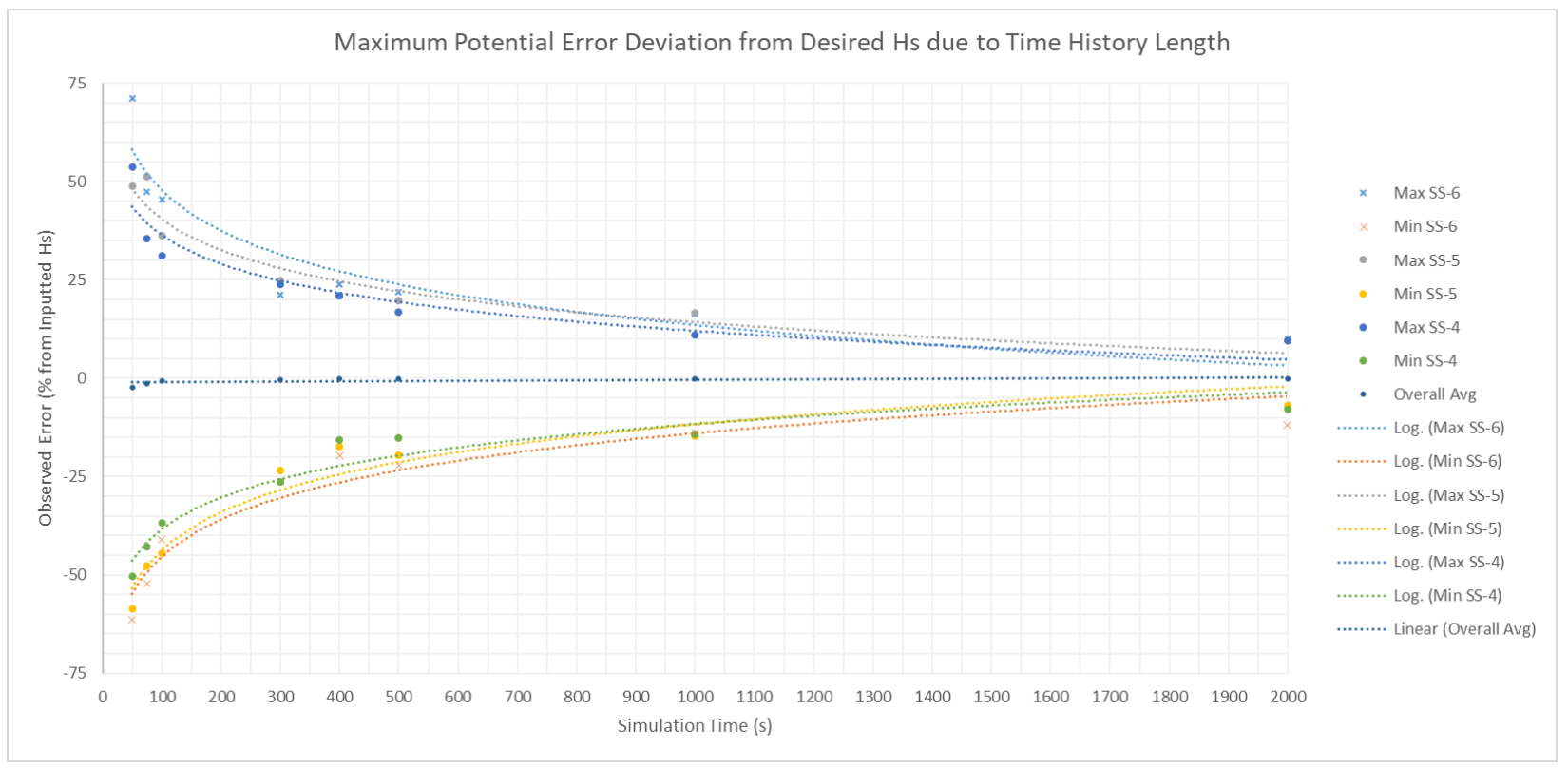

Figure 18 Combined theoretical error margin plot with respect to MATLAB simulation time 


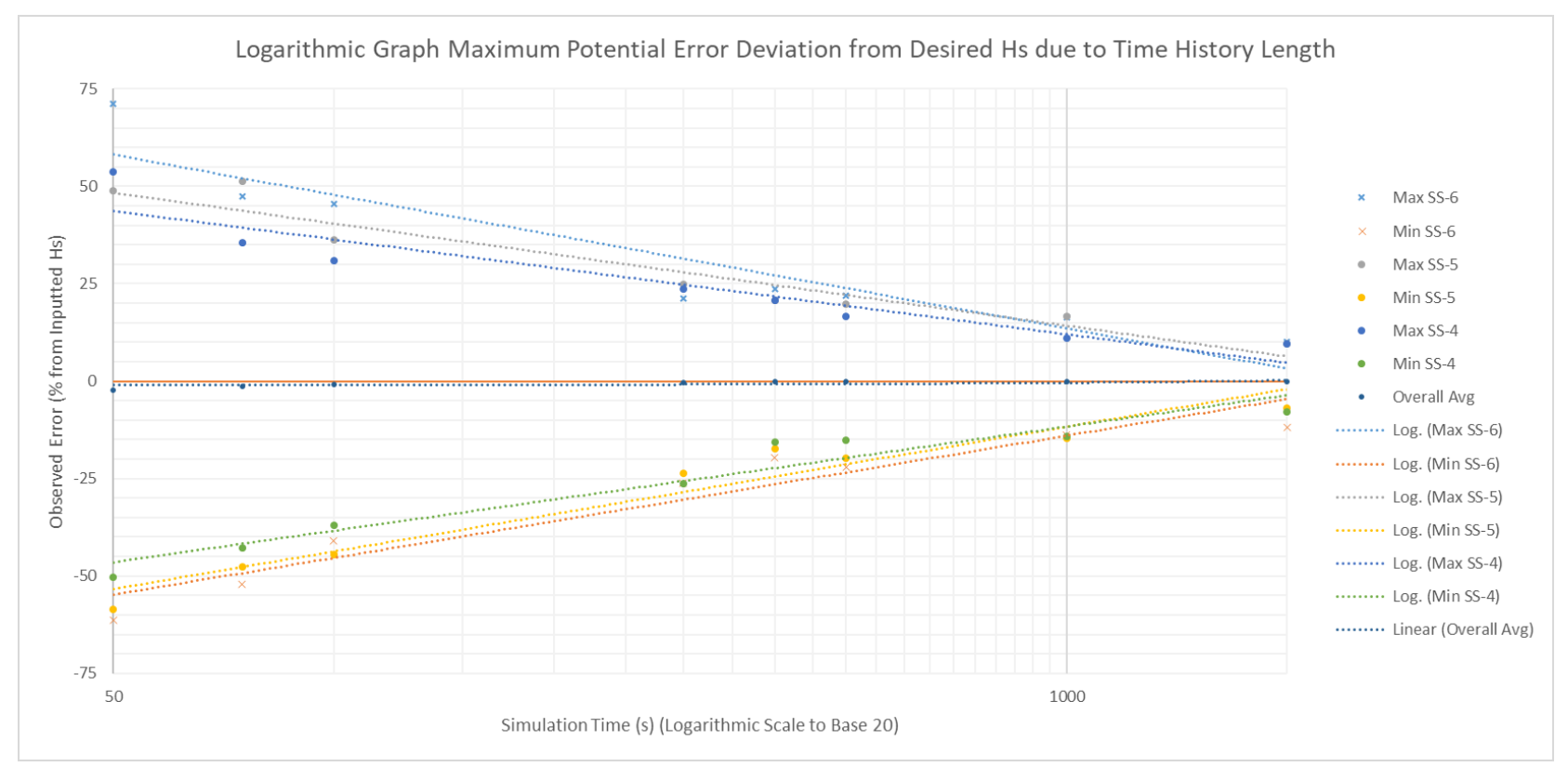

Figure 19 Logarithmic plot of combined theoretical error margins (SS-6, SS-5, SS-4) with respect to MATLAB simulation time 


\section{References}

Arena, F., Soares, C. G., \& Petrova, P. G. (2010). Theoretical Analysis of Average Wave Steepness Related to Peak Period or to Mean Period. 29th Interanational Confrence on Ocean, Offshore and Arctic Engineering (OMAE 2010). Shanghai.

Branlard, E. (2010, Febuary). Generation of tie series from spectrum: Generation of wind time series from Kaimal spectrum, Generation of wave time series from the JONSWAP spectrum. Kongens Lyngby, Denmark: Technical University of Denmark.

C.D. Simonsen, J. O. (2013). EFD and CFD for KCS heaving and pitching in regular head waves. J. Mar. Sci. Technol, 435-459.

CD-Adapco. (2017a). Deciding on K-Epsilon Model. Retrieved from file://C:/Program\%20Files/CDadapco/11.06.011/STAR-

CCM+11.06.011/doc/en/online/index.html\#page/STARCCMP\%2FGUID-C557A500-3E7A42CB-B02C-5EBDE32F0C86\%3Den\%3D.html\%23

CD-Adapco. (2017b). User guide Star-CCM+ Version 12.04.011-R8.

CD-Adapco CSP. (2017). Personal corrospondents, Customer Support Portal.

European Commission . (2013). Integrating Maritime Transport Emissions in the EU's Greenhouse Gas Reduction Policies.

Ferziger, J. H., \& Peric, M. (2002). Computational Methods for Fluid Dynamics. Springer.

ITTC. (2011a). Practical Guidelines for Ship CFD Applications. ITTC.

ITTC. (2011b). Seakeeping Experiments. International Towing Tank Conference.

Jin, Y., Chai, S., Duffy, J., Chin, C., \& Bose, N. (2017). URANS predictions of wave induced loads and motions on ships in regular head and oblique waves at zero forward speed. Jouranl of Fluids and Structures, 178-204.

Lal, A., \& Elangovan, M. (2008). CFD Simulation and Validation of Flap Type Wave-Maker.

Ming, W., Aiguo, S., Zuochao, W., Xiao, W., \& Rongrong, Y. (2013). Numerical Research on Ship Motions in Oblique Irregular Waves. Advanced Materials Research, 1531-1534.

Mousaviraad, S. M. (2010). CFD prediction of ship response to extreme winds. Iowa: University of Iowa. 
Offshore Wind. (2017, september 29). UK Offshore Wind Generation on the Rise in 2017 Q2. Retrieved from offshoreWIND.biz: https://www.offshorewind.biz/2017/09/29/uk-offshore-windgeneration-on-the-rise-in-2017-q2/

Rahm, S. (2015). The costly future of green shipping.

Roache, P. J. (1998). Verification of Codes and Calculations. AIAA Journal, 696-702.

Royal Institution of Naval Architects. (2018, Febuary). Best practices for wave flow simulations (pp3842). The Naval Architect. London.

Shen, Z.-r., Ye, H.-X., \& Wan, D.-c. (2014). URANS Simulation of ship motion responses in longcrested irregular waves. Journal of Hydrodynamics, 436-448.

Stern, F., Wilson, R. V., Coleman, H., \& Paterson, E. G. (2001). Comprehensive approach to verification and validation of CFD simulations - Part 1: Methodology and procedures. Jouranl of Fluids Engineering-Transcriptions of Asme, , 793-802.

Stern, F., Wilson, R., \& Shao, J. (2006). Quantitative V\&V of CFD simulations and certification of CFD codes. International Journal of Numerical Methods in Fluids, 50 , 1335-1355.

Terziev, M., Tezdogan, T., Oguz, E., Gourlay, T., Demirel, Y. K., \& \& Incecik, A. (2018). Numerical investigation of the behaviour and performance of ships advancing through restricted shallow waters. Journal of Fluids and Structures, 76, 185-215.

Tezdogan, T., Incecik, A., \& Turan, O. (2016). Full-scale unsteady RANS simulations of vertical ship motions in shallow water. Ocean Engineering, 131-145.

Y.H. Ozdemir, B. B. (2014). Numerical and experimental study of turbulent free surface flow for a fast ship model. Brodogradnja Volume 65 Issue 1, pp. 39-54. 